\title{
SPLIT, CHARACTERISTIC BASED SEMI-IMPLICIT ALGORITHM FOR LAMINAR/TURBULENT INCOMPRESSIBLE FLOWS
}

\author{
O. C. ZIENKIEWICZ, B. V. K. SATYA SAI, K. MORGAN AND R. CODINA \\ Institute for Numerical Methods in Engineering, University of Wales, Swansea SA2 8PP. U.K.
}

\begin{abstract}
SUMMARY
In an earlier paper, Zienkiewicz and Codina (Int. j. numer. methods fluids, 20, 869-885 (1995)) presented a general algorithm for the solution of both compressible and incompressible Navier-Stokes equations. The algorithm, based on operator splitting, permits arbitrary interpolation functions to be used while avoiding the Babŭska-Brezzi restriction. In addition, its characteristic based approach introduces a form of rational dissipation. Zienkiewicz et al. (Int. j. numer. methods fluids, 20, 887-913 (1995)) presented the application of this algorithm in its fully explicit form to various inviscid compressible flow problems. They also presented two incompressible flow problems solved by the fully explicit form, employing a pseudo compressibility. The present work deals with the application of the above algorithm it its semi-implicit form to some incompressible flow benchmark problems. Further, it extends the methodology to turbulent flows by employing both one, and two equation turbulence models. A comparison of results with earlier investigations is presented. Other issues addressed in this study include the effect of additional diffusion terms present in the scheme for both laminar and turbulent flow problems and some practical difficulties associated with local time stepping.
\end{abstract}

KEY WORDS: operator-splitting; general algorithm; characteristic-Galerkin; laminar/turbulent; incompressible

\section{INTRODUCTION}

The development of Galerkin finite element procedures for the incompressible Navier-Stokes equations can be dated back to the 1970s, when Hood and Taylor ${ }^{1}$ advocated the use of mixed interpolation with interpolation functions for pressure being at least one order less than those used for velocities. Equal-order interpolation gives rise to spurious pressure modes as a result of zero diagonal terms in the discrete steady state equations which prove singular, thus violating the Babŭska-Brezzi (B-B) restriction. Penalty forms avoid this difficulty by introducing a small non-zero term in the diagonal, but they are not robust and are doomed to fail unless the B-B condition is satisfied a priori, as is the case with certain kinds of reduced integration. ${ }^{2}$ Thus alternative stabilization methods are desirable. Hughes et al. ${ }^{3}$ have shown that such stabilization could be achieved by the addition of terms corresponding to those arising from the application of a least squares method. Sani et al..$^{4.5}$ provided some more suggestions to deal with checkerboard pressure modes and on the use of a penalty formulation without reduced integration for the penalty term. However, the same stabilization could be achieved in a natural way in many time-stepping formulations when the steady state solution is reached. The fractional step method, devised originally by Chorin ${ }^{6}$ in a finite difference context, 
forms the basis for many of the finite element formulations ${ }^{7-10}$ that employ operator splitting. The method essentially separates the pressure calculation into one involving a Laplacian form which is self-adjoint. This method, when applied to obtain the steady state solution, can in certain forms circumvent the B-B restriction, as observed by Schneider et al ${ }^{7}$ and Kawahara and Ohmiya ${ }^{8}$ and clearly explained by Zienkiewicz and $\mathrm{Wu} .{ }^{11}$

The treatment of convection-dominated flows poses another difficulty in the simulation of incompressible flows. The application of the conventional Galerkin finite element method to a scalar convection dominated problem is equivalent to central differencing of the convection terms, which causes 'wiggles' or complete instabilities to appear in the flow variables. Thus the traditional Galerkin finite element method is no longer optimal when convection phenomena dominate the flow. A possible way of dealing with wiggles is to refine the mesh in convection dominated regions. ${ }^{12} \mathrm{~A}$ more economical approach is to use upwind differences or, more generally, a suitable Petrov-Galerkin method, which not only suppresses the oscillations but also improves the accuracy of computation. Originally proposed by Christie et al., ${ }^{13}$ the upwind finite element method was later pursued and improved upon by Heinrich et al., ${ }^{14}$ Hughes and Brookes, ${ }^{15}$ Kelly et al. ${ }^{16}$ and Johnson et al. ${ }^{17}$

Another, more rational, way to obtain the upwinding effect in scalar problems is to use a characteristic based time-marching scheme, ${ }^{18}$ which provides an effective time-stepping procedure for transient solutions as well as an iterative procedure for steady state solutions. This concept forms the basis for the development of a general algorithm based on operator-splitting to deal with both the compressible and incompressible Navier-Stokes equations. ${ }^{19}$

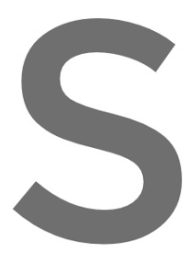

In the present work with turbulent incomp additional dissipation based formulation. A could be obtained with could lead to an under this genual algosithm in its semi-implicit form is tsed and extonted
essible flows. It is also intencied in the present work to bying out th
erms (which contribute to the upwinding effect) present in the cha
eneral observation by earlier investigators is that while oscillation
upwinding for laminar fiow problems, for turbulent predictions, do to deal
are effect of
free results
upwinding lengths. ${ }^{20}$ We find that this assertion is not generally true and that the present algorithm yields good

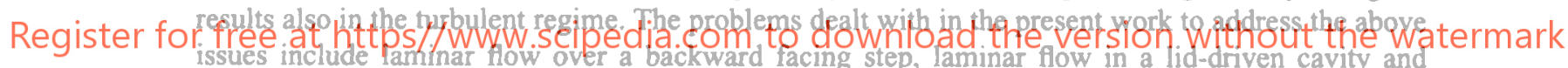
turbulent flow over a backward facing step.

A rigorous mathematical derivation of the algorithm is presented in Reference 19. Here we present the extension of this scheme to turbulence modelling using both one and two equation models of turbulence.

\section{EQUATIONS OF FLOW}

The governing equations for a Newtonian, incompressible viscous flow are written as

$$
\begin{aligned}
\nabla \cdot \mathbf{u} & =0 \quad \text { (continuity), } \\
\frac{\partial \mathbf{u}}{\partial t} & =\nabla \cdot\left[-(\mathbf{u} \otimes \mathbf{u})-P \mathbf{I}+\tau+\mathbf{f}^{\mathbf{e}}\right] \quad \text { in } \Omega \times(0, T) \text { where } \Omega \subset \mathbb{R}^{2} \quad \text { (momentum), }
\end{aligned}
$$

where $\mathrm{u}$ is the vector of velocities $\left(U_{1}, U_{2}\right), P$ is the pressure divided by the density $(p / \rho), I$ is the unit tensor, $\boldsymbol{\tau}$ is the shear stress tensor divided by the density, $f_{e}$ is the body force vector.

The shear stress tensor (divided by the density) $\tau$ is given by

$$
\tau_{i j}=v\left(\frac{\partial U_{i}}{\partial x_{j}}+\frac{\partial U_{j}}{\partial x_{i}}-\frac{2}{3} \nabla \cdot \mathbf{u} \delta_{i j}\right), \quad i, j=1,2 .
$$


Of course, the third term within the brackets on the right-hand side of this equation becomes equal to zero by virtue of equation (1).

For turbulent flows the Reynolds-averaged Navier-Stokes equations are derived by assuming that any flow variable $\phi$ can be written as

$$
\phi=\bar{\phi}+\phi^{\prime}
$$

where $\bar{\phi}$ is the mean turbulent value and $\phi^{\prime}$ is the fluctuating component. Then the Reynoldsaveraged Navier-Stokes equations become

$$
\begin{aligned}
\nabla \cdot \overline{\mathbf{u}} & =0 \quad \text { (continuity), } \\
\frac{\partial \overline{\mathbf{u}}}{\partial t} & =\nabla \cdot\left[-(\overline{\mathbf{u}} \otimes \overline{\mathbf{u}})-\bar{P} \mathbf{I}+\boldsymbol{\tau}^{\mathrm{V}}+\boldsymbol{\tau}^{\mathrm{R}}+\mathbf{f}_{\mathrm{e}}\right] \quad \text { (momentum). }
\end{aligned}
$$

Here $\tau^{\mathrm{V}}$ is the viscous shear stress tensor divided by the density, as defined by equation (3), and $\tau^{\mathrm{R}}$ is the Reynolds shear stress tensor divided by the density, defined as

$$
\tau^{\mathbb{R}}=-\overline{u^{\prime} \otimes u^{\prime}}
$$

or, in Cartesian co-ordinates,
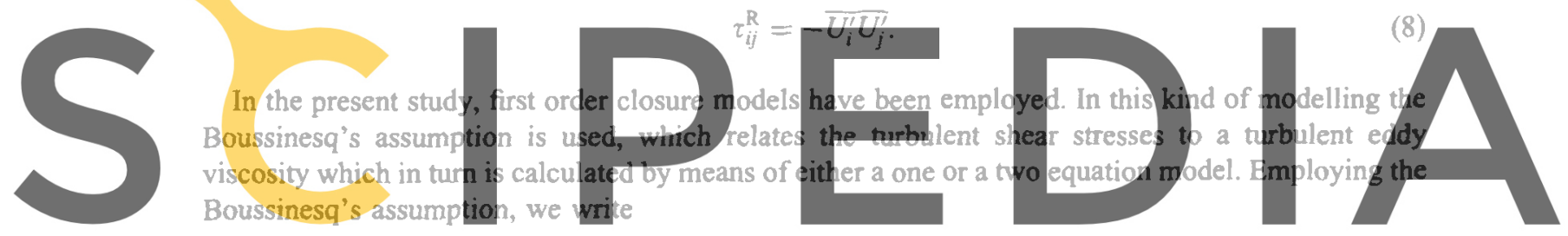

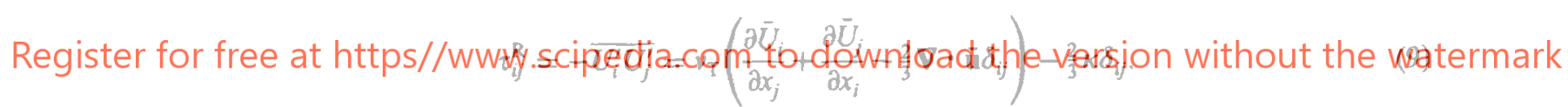

Here $v_{T}$ is the turbulent eddy kinematic viscosity coefficient and $x$ is the turbulent kinetic energy given by $\frac{1}{2}\left(\mathbf{u}^{\prime}\right)^{2}$. The momentum equation becomes

$$
\frac{\partial \overline{\mathbf{u}}}{\partial t}=\boldsymbol{\nabla} \cdot\left[-(\overline{\mathbf{u}} \otimes \overline{\mathbf{u}})-\bar{P} \mathbf{I}+\boldsymbol{\tau}^{\top}+\mathbf{f}_{\mathbf{e}}\right]
$$

where

$$
\boldsymbol{\tau}^{T}=\boldsymbol{\tau}^{\mathrm{V}}+\boldsymbol{\tau}^{\mathrm{R}}=\left(v+v_{\mathrm{T}}\right)\left(\frac{\partial \bar{U}_{i}}{\partial x_{j}}+\frac{\partial \bar{U}_{j}}{\partial x_{i}}-\frac{2}{3} \nabla \cdot \overline{\mathbf{u}} \delta_{i j}\right)-\frac{2}{3} \kappa \delta_{i j}
$$

To close the system of equations, the Prandt-Kolmogorov relationship

$$
v_{T}=c_{\mu}^{\prime} \kappa^{1 / 2} L
$$

is used, where $\kappa^{1 / 2}$ is a velocity scale for large scale turbulent motion (indeed, $\kappa$ is the turbulent kinetic energy), $L$ is a length scale for turbulence and $c_{\mu}^{\prime}$ is a constant. In the present study both one and two equation models have been used, which differ in the way in which equation (12) is evaluated. 


\subsection{One equation model}

An additional transport equation is solved for $\kappa$, the turbulent kinetic energy, while a simple algebraic relation is used for $L$, the length scale. The equation for $\kappa$ is of the form

$$
\frac{\partial \kappa}{\partial t}=-\boldsymbol{\nabla} \cdot(\kappa \tilde{\mathbf{u}})+\boldsymbol{\nabla} \cdot\left(v_{\kappa} \boldsymbol{\nabla} \kappa\right)+\left(\boldsymbol{\tau}^{\mathrm{R}} \cdot \boldsymbol{\nabla}\right) \cdot \overline{\mathbf{u}}-\epsilon .
$$

The first term on the right hand side is the convective component, the second term is the diffusion component, the third term is the turbulence production term and the fourth term is the dissipation term. Also,

$$
v_{\kappa}=v+\frac{v_{\mathrm{T}}}{\sigma_{\kappa}}
$$

where $\sigma_{\kappa}$ is a constant taken generally as unity. Further,

$$
\epsilon=C_{\mathrm{D}} \frac{\kappa^{3 / 2}}{L}
$$

where $C_{\mathrm{D}}$ is a constant. The turbulence length scale $L$ and the mixing length, $l_{\mathrm{m}}$ are related by the expression $^{21}$
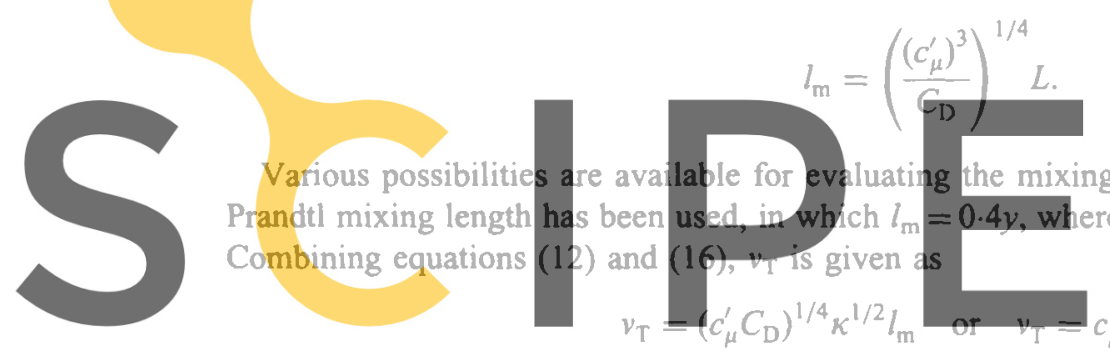

\section{Register for where $c_{\text {at }}$ is a constant taken generally as 0.09}

\subsection{Two equation model}

Here the transport equation

$$
\frac{\partial \epsilon}{\partial t}=-\nabla \cdot(\epsilon \overline{\mathbf{u}})+\nabla \cdot\left(v_{\epsilon} \nabla \epsilon\right)+C_{\epsilon 1} \frac{\epsilon}{\kappa}\left(\tau^{R} \cdot \nabla\right) \cdot \overline{\mathbf{u}}-C_{\epsilon 2} \frac{\epsilon^{2}}{\kappa}
$$

is used to evaluate $\epsilon$ where $C_{\epsilon\}}$ is a constant taken in the range $1.45-1.55$ and $C_{\epsilon 2}$ is a constant in the range $1 \cdot 92-2 \cdot 00$. Also,

$$
v_{\epsilon}=v+\frac{v_{\mathrm{I}}}{\sigma_{\epsilon}}
$$

where $\sigma_{\epsilon}$ is a constant taken equal to 1.3 .

In two equation models, $v_{\mathrm{T}}$ is evaluated by the formula

$$
v_{\mathrm{T}}=c_{\mu} \frac{\kappa^{2}}{\epsilon} \text {. }
$$

Frequently the initial and boundary conditions for the above models are taken in the form $\kappa$ and $\epsilon$ given at $t=0$,

$$
\left.\kappa\right|_{\Gamma}=0,\left.\quad \epsilon\right|_{\Gamma}=\epsilon_{\mathrm{b}},
$$


where $\Gamma$ is a solid boundary. However, in general, $\epsilon_{b}$ is not known. Further, these models are not valid near walls where the local $R e$ is too small. To account for the near-wall effects, either wall functions or low Reynolds number versions have to be used. In the present analysis, low Reynolds number versions have been employed for both one and two equation models. This simply involves a modification of various coefficients of the original models by multiplying them by suitable damping functions.

\subsection{Low Re version of the one equation model}

Wolfstein ${ }^{22}$ suggested the modifications

$$
\begin{aligned}
v_{\mathrm{t}} & =c_{\mu}^{1 / 4} \kappa^{1 / 2} l_{\mathrm{m}} f_{\mu}, \\
\epsilon & =C_{D} \frac{\kappa^{3 / 2}}{L f_{b}},
\end{aligned}
$$

where

$$
f_{\mu}=1-\mathrm{e}^{-0.160 R_{\mathrm{k}}}, \quad f_{\mathrm{b}}=1-\mathrm{e}^{-0.263 R_{\mathrm{k}}}, \quad R_{\mathrm{k}}=\sqrt{\kappa} \frac{y}{v}
$$

and $y$ is the distance from the nearest wall.
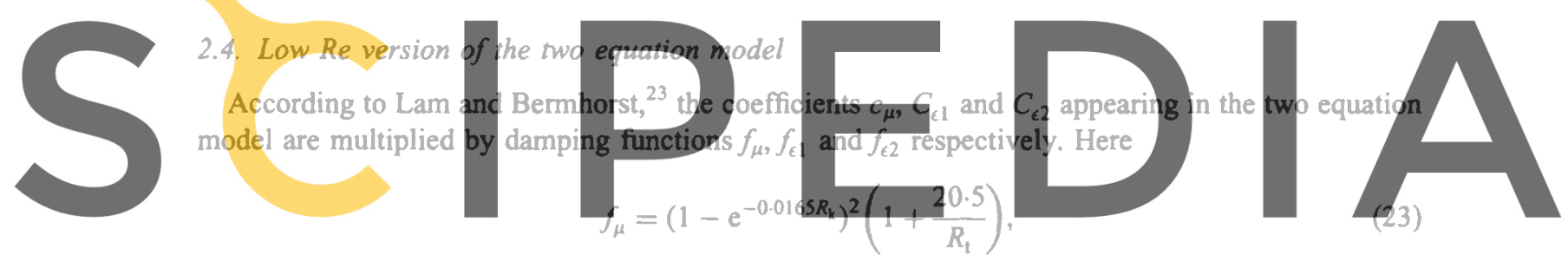

\section{Register for free at https//www.scipedia.com to dow oł ${ }^{3}$ ad the version without the watermark

$$
f_{c 2}=1-\mathrm{e}^{-R_{1}^{2}}
$$

where $R_{\mathrm{t}}=\kappa^{2} / v \epsilon$. The wall boundary conditions are now $\kappa=0$ and $\partial \epsilon / \partial y=0$.

\section{SOLUTION METHODOLOGY}

Reverting to the notation used in Reference 19 and dropping the overbar for turbulent averaged quantities for convenience, the full system of equations can be represented as

$$
\frac{\partial V}{\partial t}+\frac{\partial F_{i}}{\partial x_{i}}+\frac{\partial G_{i}}{\partial x_{i}}+Q=0
$$

where

$$
V^{\mathrm{T}}=\left[0, U_{1}, U_{2}, \kappa, \epsilon\right]
$$

is the independent variable vector (note that $\epsilon$ does not appear for the one-equation model),

$$
F_{i}^{\mathrm{T}}=\left[U_{i}, U_{1} U_{i}+\delta_{1 i}\left(P+\frac{2}{3} \kappa\right), U_{2} U_{i}+\delta_{2 i}\left(P+\frac{2}{3} \kappa\right), U_{i} \kappa, U_{i} \epsilon\right]
$$


is the convective flux vector,

$$
G_{i}^{\mathrm{T}}=\left[0,-\tau_{1 i}^{\mathrm{T}},-\tau_{2 i}^{\mathrm{T}},-\left(v+\frac{v_{\mathrm{T}}}{\sigma_{\kappa}}\right) \frac{\partial \kappa}{\partial x_{i}},-\left(v+\frac{v_{\mathrm{T}}}{\sigma_{\mathrm{\epsilon}}}\right) \frac{\partial \epsilon}{\partial x_{i}}\right]
$$

is the viscous flux vector and

$$
Q^{T}=\left[0, g_{1}, g_{2},-\tau_{i j}^{\mathrm{R}} \frac{\partial U_{i}}{\partial x_{j}}+\epsilon,-C_{\epsilon 1} \frac{\epsilon}{\kappa} \tau_{i j}^{\mathrm{R}} \frac{\partial U_{i}}{\partial x_{j}}+C_{\epsilon 2} \frac{\epsilon^{2}}{\kappa}\right] .
$$

The various steps involved in the discretization of such equations have been explained in Reference 19. Here we only present a summary of the steps and the extension to the incompressible $\kappa$ and $c$ equations. In all the ensuing equations the standard Galerkin procedure is used with the discretization

$$
\begin{aligned}
& U_{i}=N \bar{U}_{i}, \quad \Delta U_{i}=N \Delta \bar{U}_{i}, \quad \Delta \tilde{U}_{i}=N \Delta \tilde{\bar{U}}_{i}, \quad P=N_{\mathrm{p}} \bar{P}, \\
& \kappa=N \bar{\kappa}, \quad \epsilon=N \bar{\epsilon}, \quad \Delta \kappa=N \Delta \bar{\kappa}, \quad \Delta \varepsilon=N \Delta \bar{\epsilon},
\end{aligned}
$$

where an overbar denotes the finite element nodal values.
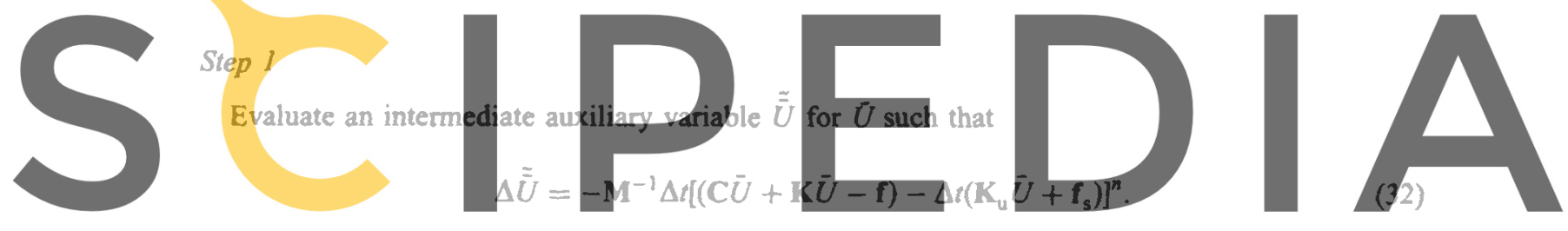

The matrices in the above equation are evaluated as

Register for free at https//Www.scipedia.com to download the version without the watermark

$$
\begin{aligned}
M & =\int_{\Omega} N^{\mathrm{T}} N \mathrm{~d} \Omega, \quad \mathrm{C}=\int_{\Omega} N \frac{\partial\left(\bar{U}_{i} N^{\mathrm{T}}\right)}{\partial x_{i}} \mathrm{~d} \Omega, \\
\mathbf{K} & =\int_{\Omega}\left(v+v_{\mathrm{T}}\right) \frac{\partial N^{\tau}}{\partial x_{i}} \frac{\partial N}{\partial x_{i}} \mathrm{~d} \Omega+\text { boundary terms, } \quad \mathbf{f}=\int_{\Omega} N^{\mathrm{T}} \frac{2}{3} \frac{\partial(N \bar{\kappa})}{\partial x_{i}} \mathrm{~d} \Omega, \\
\mathbf{K}_{\mathrm{u}} & =\frac{1}{2} \int_{\Omega} \frac{\partial}{\partial x_{i}}\left(\bar{U}_{i} N^{\mathrm{T}}\right) \frac{\partial}{\partial x_{j}}\left(\bar{U}_{j} N\right) \mathrm{d} \Omega, \quad \mathbf{f}_{\mathrm{s}}=-\frac{1}{2} \int_{\Omega} \frac{\partial}{\partial x_{i}}\left(\bar{U}_{i} N^{\mathrm{T}}\right) \frac{2}{3} \frac{\partial(N \bar{\kappa})}{\partial x_{i}} \mathrm{~d} \Omega .
\end{aligned}
$$

Step 2

Solve a Poisson equation for pressure,

$$
\Delta t^{2} \theta_{1} \theta_{2} \mathbf{H} \Delta \bar{P}=\Delta t\left[\mathbf{Q}\left(\bar{U}+\theta_{1} \Delta \tilde{\bar{U}}\right)-\Delta t \theta_{1} \mathbf{H} \bar{P}-\mathbf{f}_{\mathrm{p}}\right]^{n}
$$

where

$$
\mathbf{H}=\int_{\Omega} \frac{\partial N_{\mathrm{p}}^{\mathrm{T}}}{\partial x_{i}} \frac{\partial N_{\mathrm{p}}}{\partial x_{i}} \mathrm{~d} \Omega . \quad \mathbf{Q}=\int_{\Omega} \frac{\partial N_{\mathrm{p}}^{\mathrm{T}}}{\partial x_{i}} N \mathrm{~d} \Omega .
$$


Step 3

Correct the velocity via

$$
\begin{gathered}
\Delta \bar{U}=\Delta \tilde{\bar{U}}-\mathbf{M}^{-1} \Delta t\left[\mathbf{Q}^{\mathrm{T}}\left(\bar{P}+\theta_{2} \Delta \bar{P}\right)+\Delta t \mathbf{P} \bar{P}\right]^{n}, \\
\mathbf{P}=\frac{1}{2}\left(1-\theta_{2}\right) \int_{\Omega} \frac{\partial}{\partial x_{2}}\left(\bar{U}_{i} N_{\mathrm{p}}^{\mathrm{T}}\right) \frac{\partial N_{\mathrm{p}}}{\partial x_{i}} \mathrm{~d} \Omega,
\end{gathered}
$$

where $\theta_{1}$ and $\theta_{2}$ are integration parameters and $\theta_{1}, \theta_{2} \in[0,1]$.

\section{Step 4}

\section{Using $\bar{U}$ and $\bar{P}$, evaluate $x$ in the one equation model or both $x$ and $\epsilon$ in the two equation model} as

$$
\Delta \bar{\kappa}=-\Delta t\left[\mathbf{C} \bar{\kappa}+\mathbf{K}_{\kappa} \bar{\kappa}+\mathbf{f}_{\kappa}-\Delta t\left(\mathbf{K}_{\mathrm{u}} \bar{\kappa}+\mathbf{f}_{\kappa s}\right)\right]^{n}
$$

where
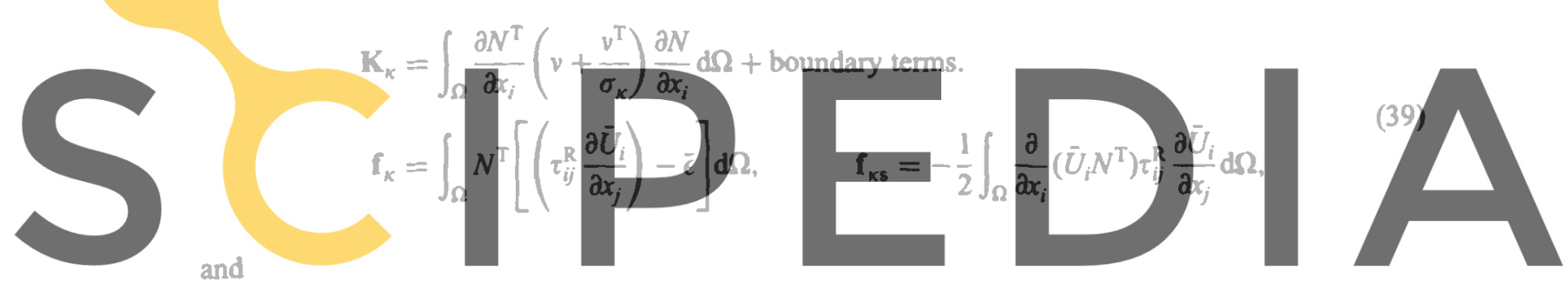

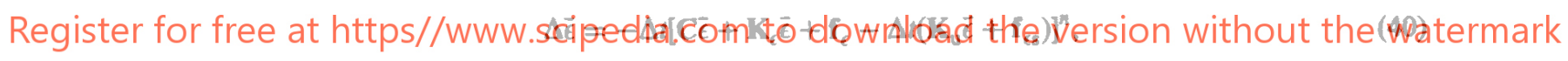

where

$$
\begin{aligned}
\mathbf{K}_{\epsilon} & =\int_{\Omega} \frac{\partial N^{\mathrm{T}}}{\partial x_{i}}\left(v+\frac{v^{\mathrm{T}}}{\sigma_{\epsilon}}\right) \frac{\partial N}{\partial x_{i}} \mathrm{~d} \Omega+\text { boundary terms, } \\
\mathbf{f}_{\epsilon} & =\int_{\Omega} N^{\mathrm{T}}\left[\left(C_{\epsilon 1} \frac{\bar{\epsilon}}{\bar{\kappa}} \tau_{i j}^{\mathrm{R}} \frac{\partial \bar{U}_{i}}{\partial x_{j}}\right)-C_{\epsilon 2} \frac{\bar{\epsilon}^{2}}{\overline{\bar{\kappa}}}\right] \mathrm{d} \Omega, \\
\mathbf{f}_{\epsilon 3} & =-\frac{1}{2} \int_{\Omega} \frac{\partial}{\partial x_{i}}\left(\bar{U}_{i} N^{\mathrm{T}}\right)\left(C_{\epsilon 1} \frac{\bar{\epsilon}}{\bar{\kappa}} \tau_{i j}^{\mathrm{R}} \frac{\partial \bar{U}_{i}}{\partial x_{j}}-C_{\epsilon 2} \frac{\bar{\epsilon}^{2}}{\bar{\kappa}}\right) \mathrm{d} \Omega .
\end{aligned}
$$

It should be noted that the treatment of the turbulent quantities brings an additional term $f$ and its associated second-order term $f_{s}$ into (32). These terms are defined in (33). Thus an additional upwinding effect is produced by the presence of $f_{s}$.

The treatment of boundary conditions in the finite element approximation is discussed in Reference 19 and in more detail in Reference 24 . In the latter reference it is shown that the conventionally used procedure of imposing $U_{i}$ on $\tilde{U}_{i}$ on solid boundaries is incorrect and a more rational procedure avoids this. In all the examples presented here, the procedure outlined in Reference 24 has been used. 


\section{CHOICE OF TIME STEP}

Equation (32) above is conditionally stable. Applying a linear stability analysis to the one dimensional convection-diffusion equation, the critical time step can be shown to be

$$
\Delta t_{\mathrm{crit}}=\frac{h}{|u|}\left[\sqrt{\left.\left(\frac{1}{P e^{2}}+1\right)-\frac{1}{P e}\right]}\right.
$$

where $h$ is a representative element length, $|u|$ is the maximum velocity in the element and $P e$ is the Peclet number, defined as

$$
P e=|u| h / v \text {. }
$$

If the time-stepping scheme is operated locally at or near the critical stability limit, the steady state solution reached will be close to that resulting from the ontimal Petrov-Galerkin process for the steady state. ${ }^{2}$ This requires that the critical time step be evaluated at each node and be used for time stepping at that node. However, on some occasions this could give rise to unrealistic differences in local time steps at different nodes in the flow domain, which could in turn result in the collapse of the computation. For example, in the wall-driven cavity problem which is presented later, near the top wall the mesh size is small and the associated velocities are large so as to give a very small time step. In contrast, at the centre of the cavity the mesh size is large and the associated velocities are small so as to give a very large time step. Another option is to choose a locally optimal value for the 'interior $\Delta t^{\prime}$ (that occurring inside the square brackets in equations $(32),(34),(36),(38)$ and $(40)$ ) and use a globally minimum value for the 'Exterior $\Delta$ '. A preliminary study on the cavity problem for various Reynolds numbers has been conducted using this methodology. However, the velocity field was observed to be damped by an excessive additional diffusion. Thus for further studies in this paper globally minimum value of the tine step was used for both internal and extenal $\Delta t$. The critical fin step, as evaluated by equations (42) and (43), is applicable for laminar flow problems only, where the diffusion terms are only those arising owing to the viscous shear stresses. For turbulent flow

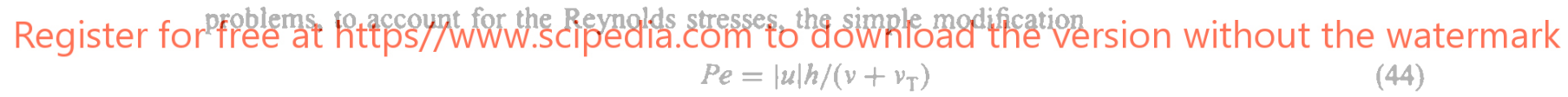

is made to equation (43). In the case of the two equation model for turbulence, the $\epsilon$ equation poses severe restrictions on the time step owing to the presence of the troublesome source term. Thus the overall time step has to be less than that predicted by equation (42). The strategies used in the present study to overcome this restriction are discussed in the next section.

\section{SOURCE TERM IN THE TWO EQUATION MODEL}

While the critical time step for laminar viscous flows is given by equation (42), for the $k-\epsilon$ model the time step is further restricted by the source term $C_{\epsilon 2} \epsilon^{2} / \kappa$. In the limit $t \rightarrow 0$ the $\kappa \rightarrow$ model is extremely 'stiff' near the wall boundaries, since $\kappa=0$ on the walls. As a result, the two-equation model requires more time steps to reach convergence than the one-equation model. One way of removing this problem is to specify initial profiles for $\kappa$ and $\epsilon$ to guarantee convergence. This is not always possible in complex flow situations. There are two possible ways of avoiding this difficulty.

1. Neglect the source term in the $\epsilon$ equation until such time that the solution develops and reasonable profiles for $\kappa$ and $\epsilon$ develop near the walls.

2. Use a fixed mixing length for the initial development ('warm-up') of the solution. This means using equation (15) for $\epsilon$ in equation (13). After a few thousand time steps the fixed mixing 
length can be turned off and $\epsilon$ can be used in equation (13). This is the method that has been used in the present work when employing the two equation model.

\section{NUMERICAL CALCULATIONS}

The following benchmark problems have been chosen to evaluate the performance of the present scheme for incompressible flows:

(a) laminar flow over a backward facing step

(b) laminar flow in a lid-driven cavity

(c) turbulent flow over a backward facing step.

For all three cases both the present scheme and the standard velocity correction scheme (standard Galerkin plus operator splitting) have been run for comparison. It may be remarked here that the standard velocity correction (SVC) scheme could be recovered from the present scheme by simply
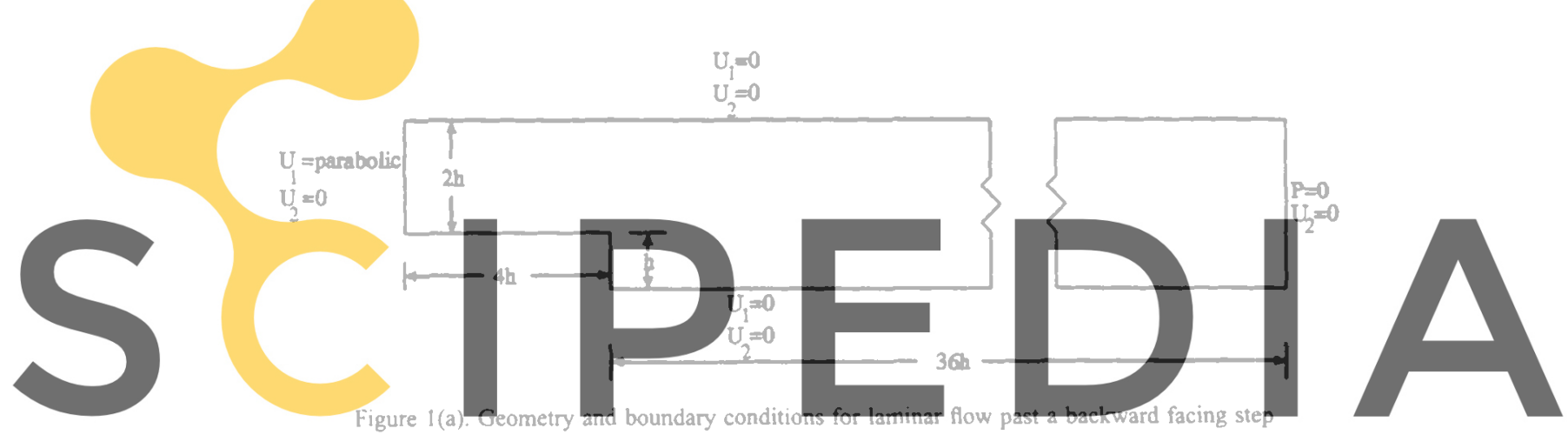

Register for free at https//www.scipedia.com to download the version without the watermark

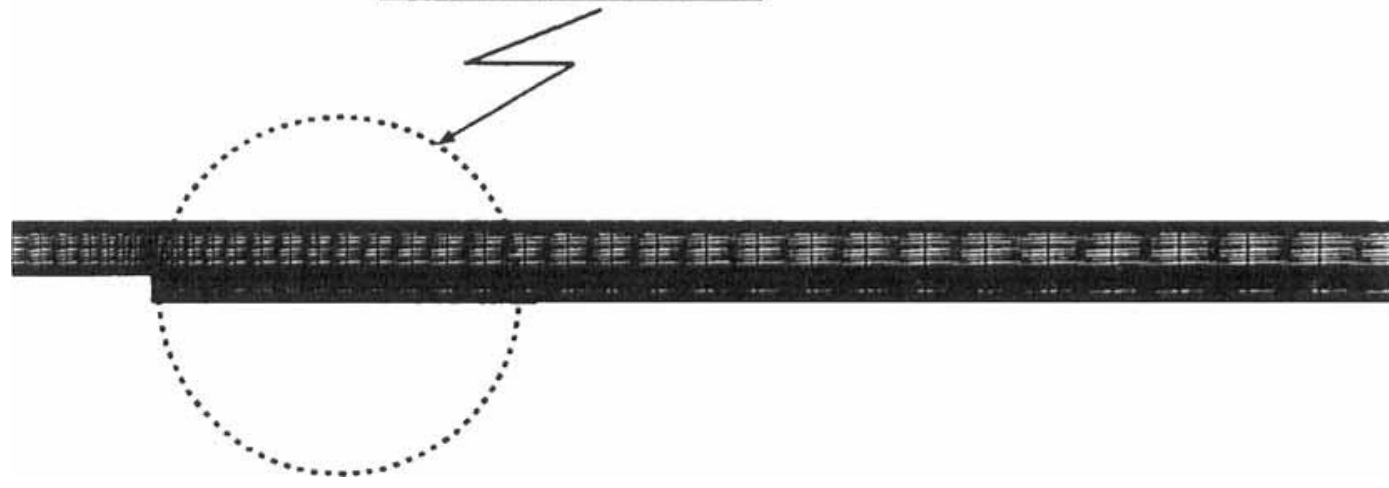

Figure 1(b). Mesh (1537 nodes, 2912 elements) for laminar flow past a backward facing step 
dropping the second-order terms (which contribute to the upwinding effect). The first two examples demonstrate the accuracy of the present scheme. Further, in the first example the present scheme gives a better prediction of reattachment lengths as compared with the SVC. Also, for both these examples the pressure field predictions are smoother than with the SVC. The third example illustrates the application of the present scheme to turbulence modelling using both the one- and two-equation models of turbulence. For all the problems, non-uniform structured meshes with linear triangular elements have been useed. A banded Gaussian solver has been used to solve the pressure Poisson equation (34).

\subsection{Laminar flow over a backward facing step}

Experimental results for this case are nrovided by Denham and Patrick ${ }^{25}$ The cases considered are $R e=73,191$ and 229. The Reynolds number is based on the average velocity at the entrance and the height of the step. The expansion ratio of the step is $2: 3$. The entry is at a distance of 4 step heights upstream of the step. The outflow boundary has been taken sufficiently far away ( 36 step heights downstream of the step) to obtain undisturbed flow conditions at the outlet. Figure l(a) shows the geometry and boundary conditions and Figure $1(b)$ shows the finite element mesh for the problem. The mesh with 1537 nodes and 2912 elements is non-uniformly spaced, with fine meshes near the wall. Figures 2(a)-2(c) show the pressure contours with the present scheme (top) and the SVC (bottom) for $R e=73,191$ and 229 iespectively. The present schene yields smoother pressure fields than the SVC. Further, meaning ful result, as ca sections behind the step Patrick $^{25}$ and the SVC results have also been between the present scheme and the SVC except in the vicinil agreement with the experimental results ${ }^{25}$ can be observed. The most common criterion to judge the performance of varjous numerical schemes for this problem is the prediction of ithe reattachment lengti behind the step. In Figure 4 a companison of the reattachment lengths predicted by the present scheme, the SVC and Taylor et $a l^{26}$ and the experimental results ${ }^{25}$ is presented. This shows that the present scheme yieids better resuits than the scheme of Taylor et al., ${ }^{26}$ who used an upwinding proposed by Heinrich et al ${ }^{14}$ Note that the reattachment length prediction by the SVC for $R e=229$ is not shown in the figure, as the solution could not be obtained for this. Table I gives the reattachment length predictions by the various methods and the percentage errors from the experimental results of Denham and Patrick. ${ }^{26}$

In general, the predictions by the present scheme appear to be better than those of Taylor et al. ${ }^{26}$ and the SVC. Finally, Figure 5 shows the streamline contours by the present scheme for the three Reynolds numbers mentioned above. The enlarged views near the step are also shown for each case.

Table I. Comparison of reattachment lengths by various methods (figures in brackets indicate percentage errors from experimental results of Denham and Patrick ${ }^{25}$ )

\begin{tabular}{rcccc}
\hline$R e$ & Denham and Patrick & Taylor et al. $^{26}$ & S-G method & Present \\
\hline 73 & 3.9 & $5.3(35.9)$ & $4.9(25.6)$ & $4.8(23.0)$ \\
191 & 8.6 & $9.4(9.3)$ & $9.7(12.7)$ & $9.2(7.0)$ \\
229 & 10.0 & $11.4(14.0)$ & - & $10.9(9.0)$ \\
\hline
\end{tabular}




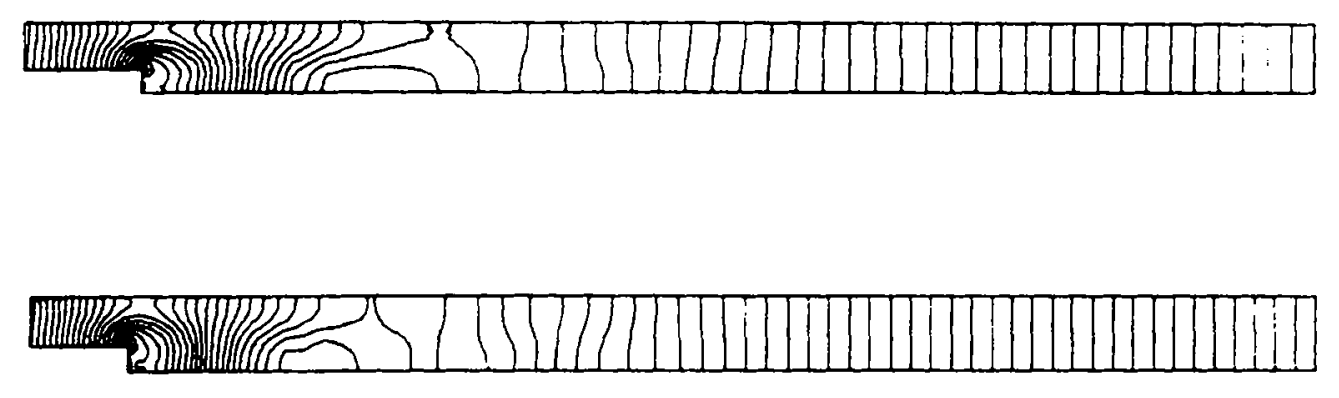

(a) $\operatorname{Re}=73$
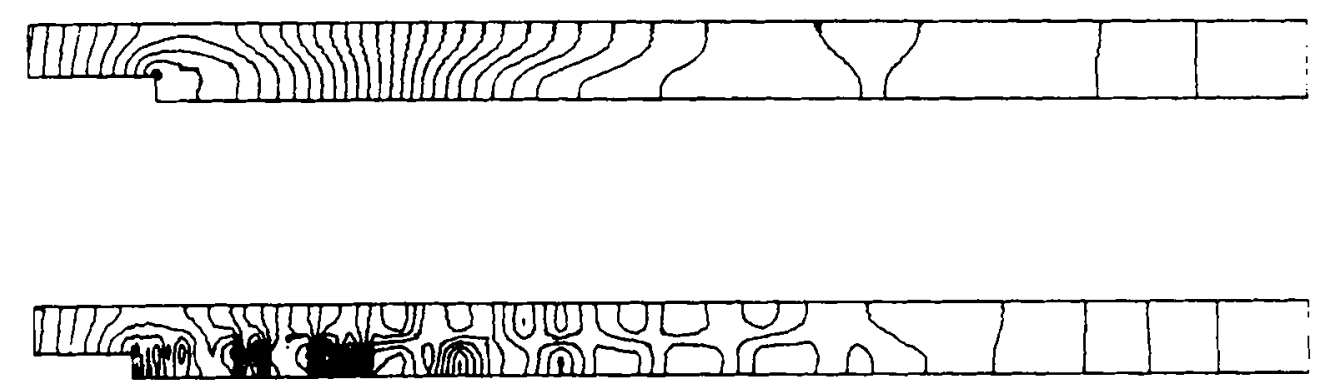

(b) $\operatorname{Re}=191$
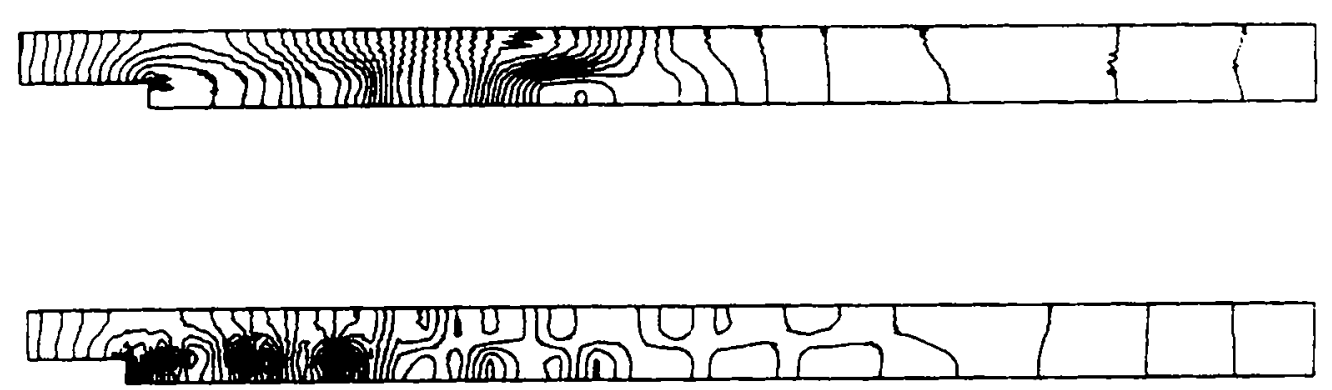

(c) $\operatorname{Re}=229$

Figure 2. Pressure contours for laminar flow past a backward-facing step: top, present scheme (upwind); bottom, SVC (no upwind) 


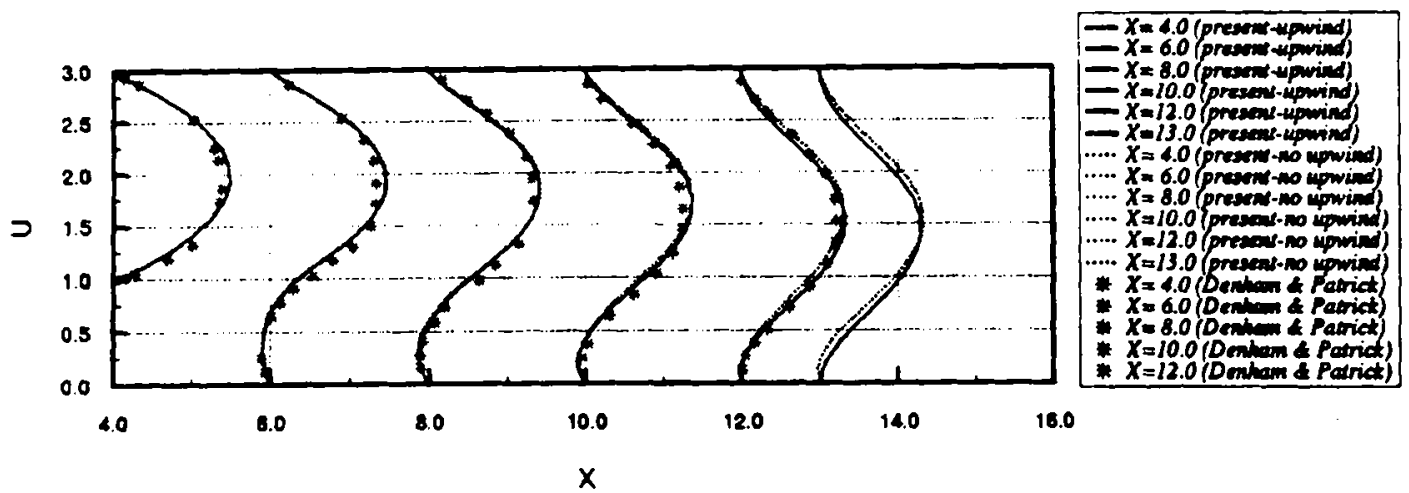

Figure 3. Velocity profiles downstream of step for $R e=191$

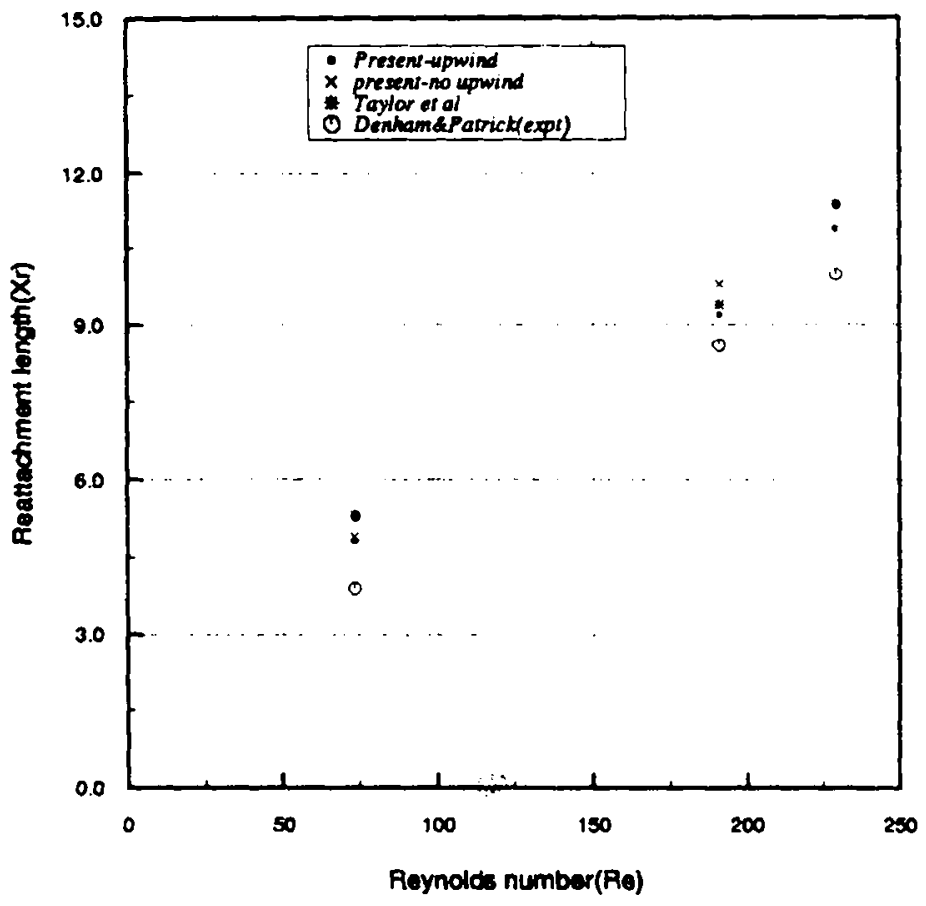

Figure 4. Comparison of reattachment lengths with various schemes

\subsection{Laminar flow in a lid-driven cavity}

Figure 6(a) shows the geometry and boundary conditions for this problem. A zero-velocity condition exists on all the walls except the top one, which moves with a (non-dimensional) horizontal velocity equal to unity. The non-dimensional pressure at the middle point of the bottom wall is fixed as zero. Figure 6(b) shows the mesh for this problem, which consists of 1521 nodes and 2888 elements. Based on some preliminary studies with two different meshes, it was observed that this mesh is sufficiently fine for the present study. The Reynolds numbers investigated are 400,1000 and 


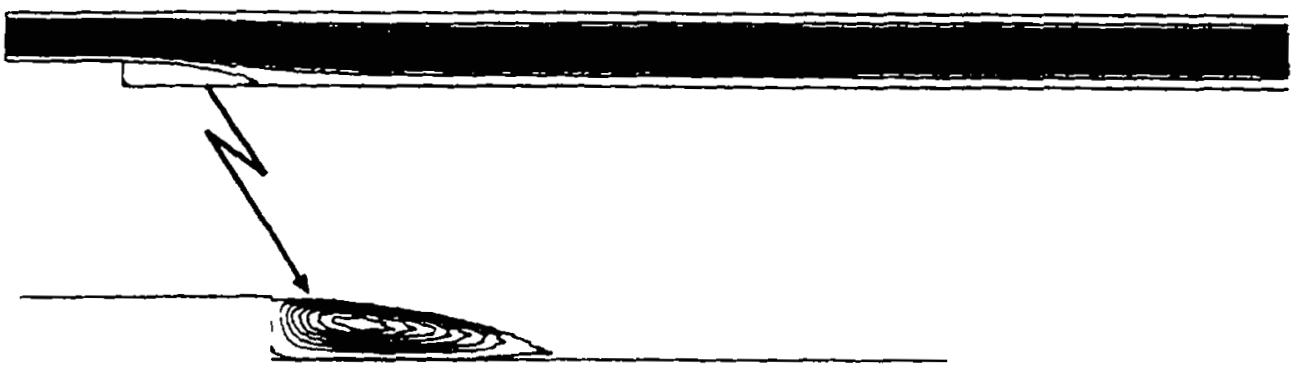

(a) $R_{e}=73$

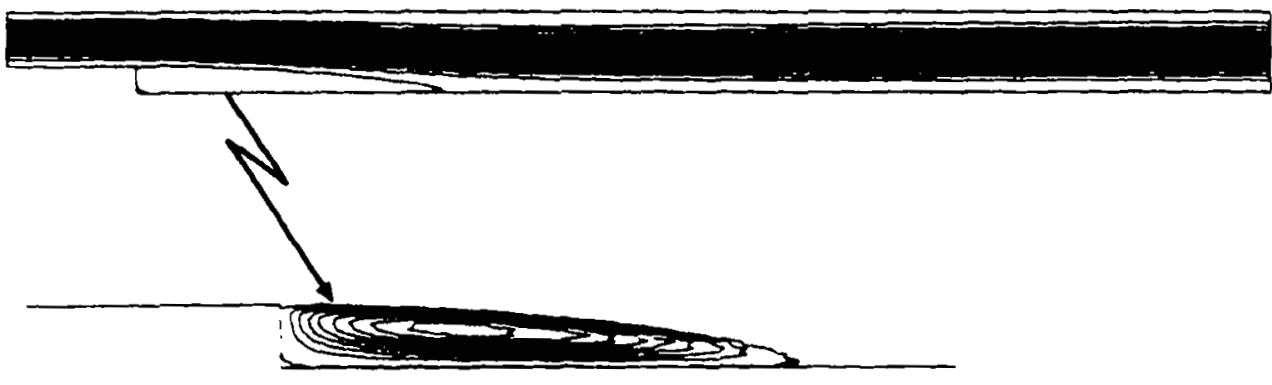

(b) $R_{e}=191$

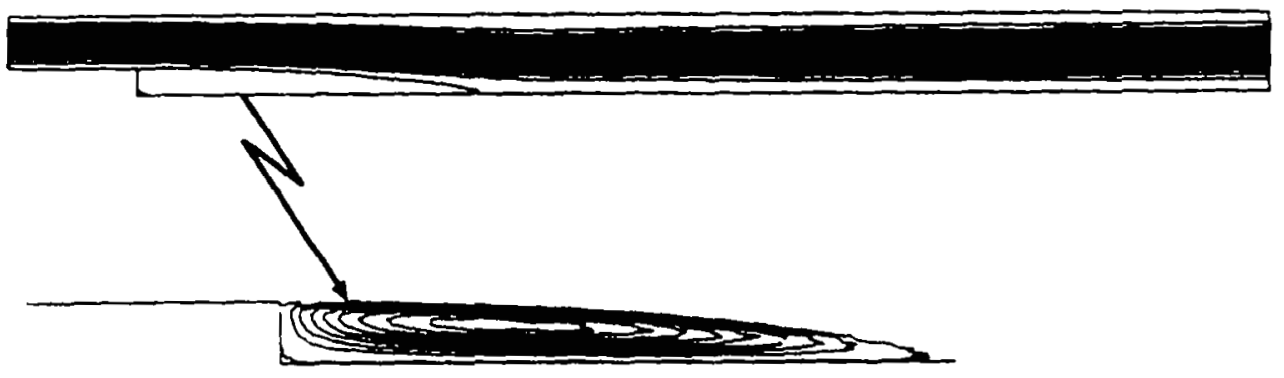

Figure 5. Streamline patterns for various Reynolds numbers with present scheme 


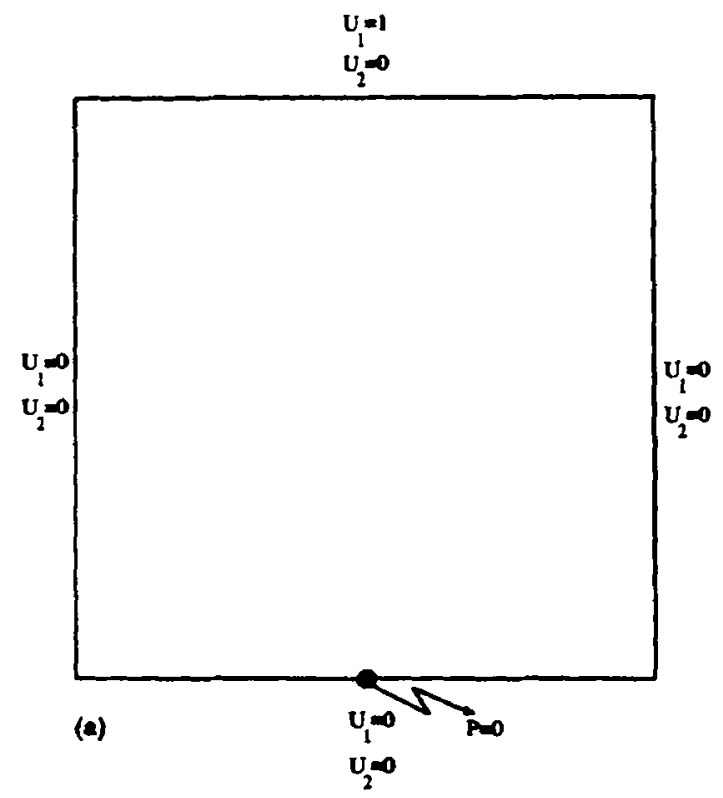

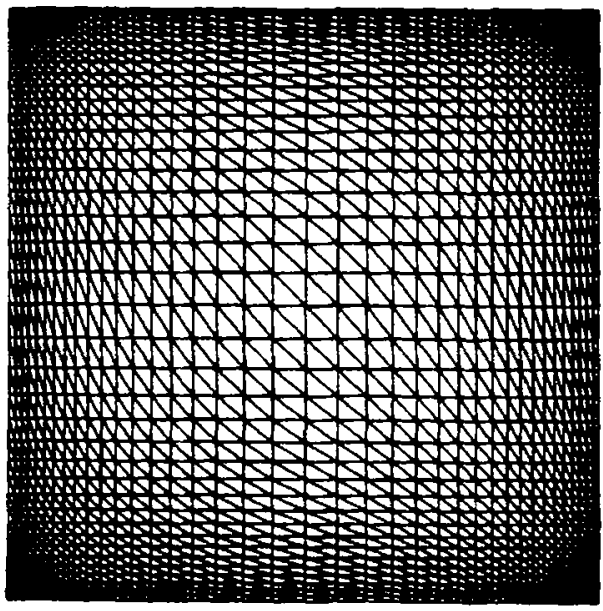

(b)

Figure 6. Laminar flow in a lid-driven cavity: (a) geometry and boundary conditions; (b) mesh (1521 nodes, 2888 elements)

5000. Once again, both the present scheme and the standard velocity correction (SVC) method have been run on this mesh. Figures 7(a)-7(c) show the pressure contours for the two schemes for $R e=400,1000$ and 5000 respectively. The left sequence shows the pressure contours with the present scheme, while the right sequence shows the same with the SVC. As expected, the pressure contours with the present scheme, especially at higher $R e$, are smoother and more wiggle-free than those with the SVC. Figure 8(a) shows the $U_{1}$-velocity profile along the mid-vertical plane for $R e=400$ with both schemes. Also plotted are the benchmark values obtained by Ghia et al. ${ }^{27}$ using a multigrid strategy with the streamfunction-vorticity formulation. From this figure it can be noticed that the present scheme and the SVC give more or less the same result. A very small amount of damping could be observed with the present scheme which can be attributed to the presence of additional diffusion terms. A somewhat different observation has been made by Sohn, ${ }^{28}$ who used a BTD-type upwinding for the same problem. In his case the upwinding resulted in a substantial amount of damping. Figures 8(b) and 8(c) show similar comparisons for $R e=1000$ and 5000 respectively. Figures 9 (a)-9(c) show the streamline patterns and Figures $10(a)-10(\mathrm{c})$ show the velocity vectors for $R e=400,1000$ and 5000 respectively with the present scheme.

The above two examples demonstrate the application of the present scheme in its semi-implicit form for laminar incompressible flow problems. While giving accurate solutions to both problems, the present scheme has also been able to provide smoother pressure fields as compared with the SVC.

\subsection{Turbulent flow over a backward facing step}

This problem is widely chosen to test the numerical performance of turbulence codes. Both one and two equation models have been used by Atkins et al. ${ }^{20}$ and Taylor et al..$^{26}$ and the two equation model has been used by Sohn, ${ }^{28}$ Hackman et al. ${ }^{29}$ and Autret $e t$ al., ${ }^{30}$ while experimental results are provided by Denham et al. ${ }^{31}$ The geometry used for this problem is similar to the one for the laminar flow problem, but the outlet is considered at 24 step heights downstream of the step. The Reynolds 


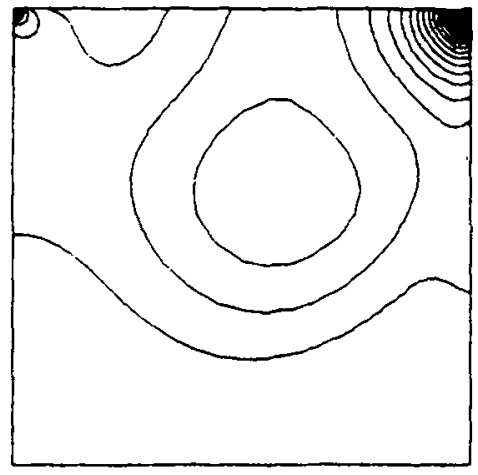

(a) $\operatorname{Re}=400$
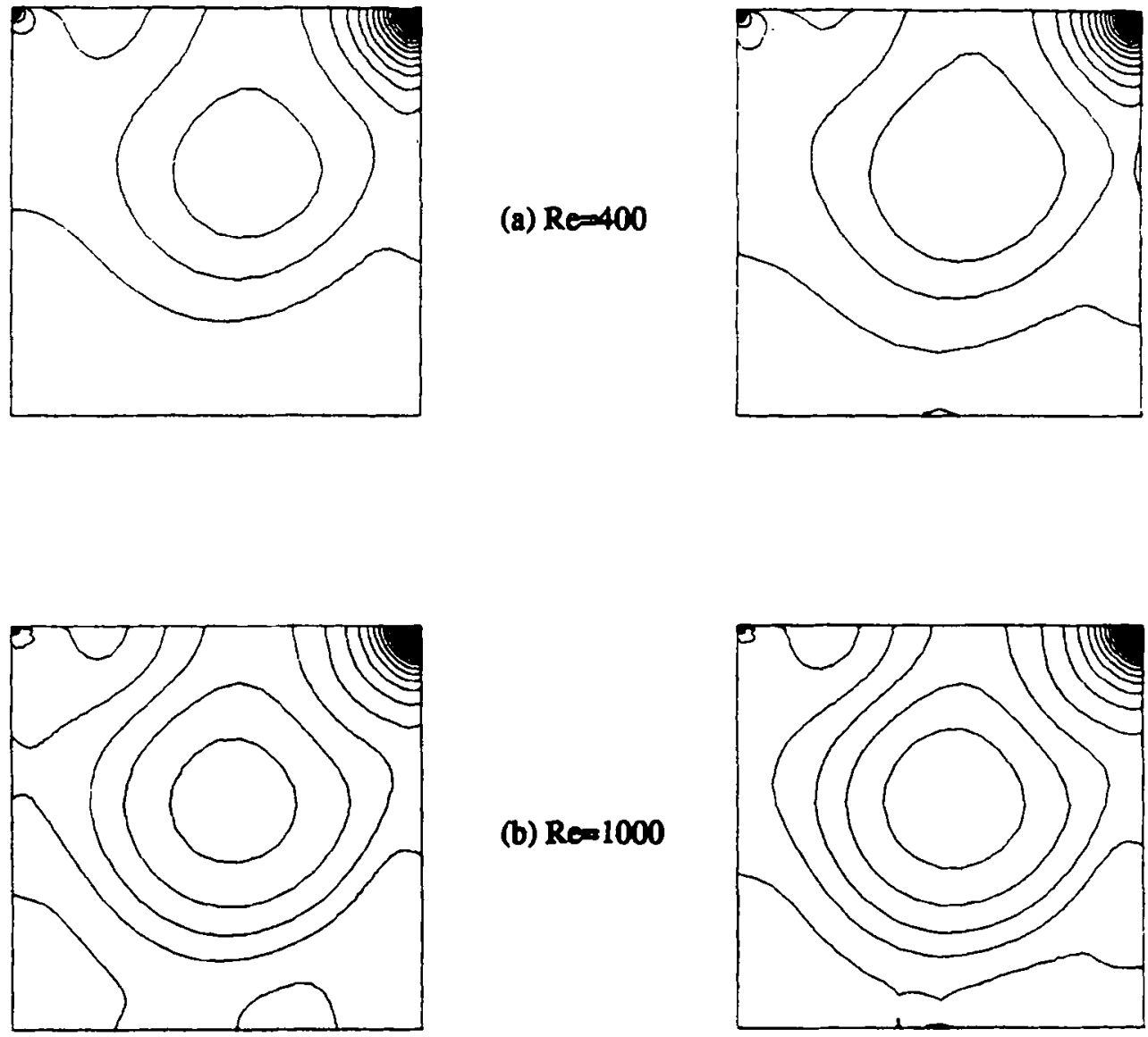

(b) Re 1000
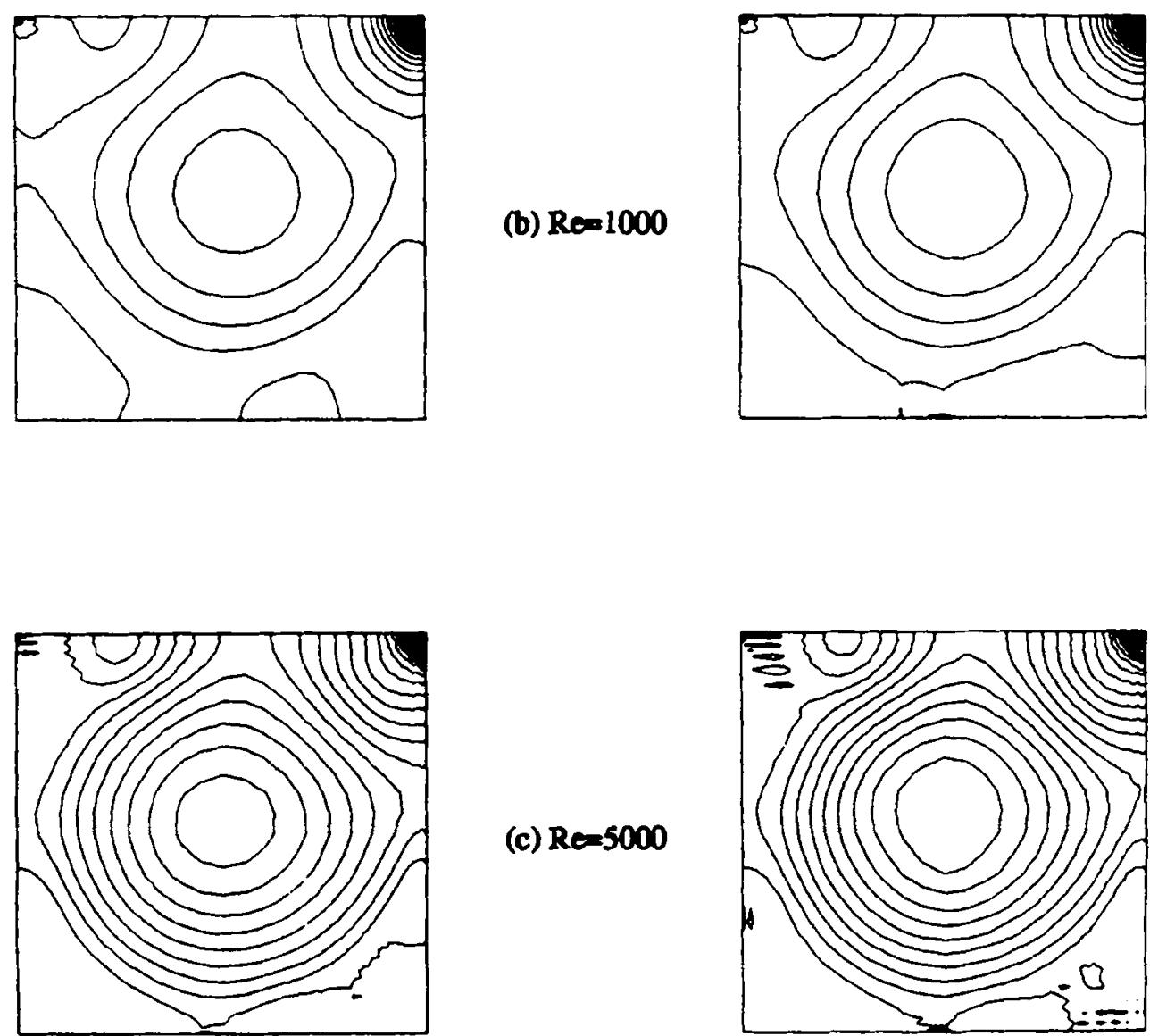

(c) Re=5000

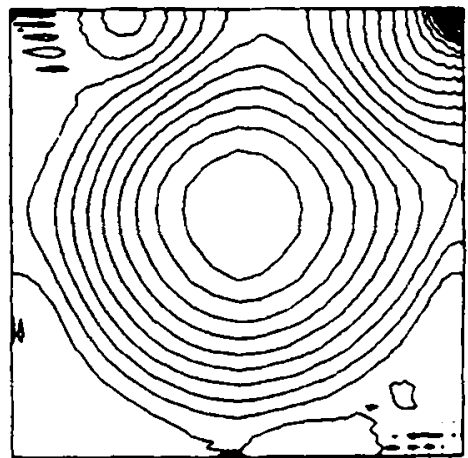

Figure 7. Pressure contours for various Reynolds numbers: left, present scheme (upwind); right, SVC (no upwind) 


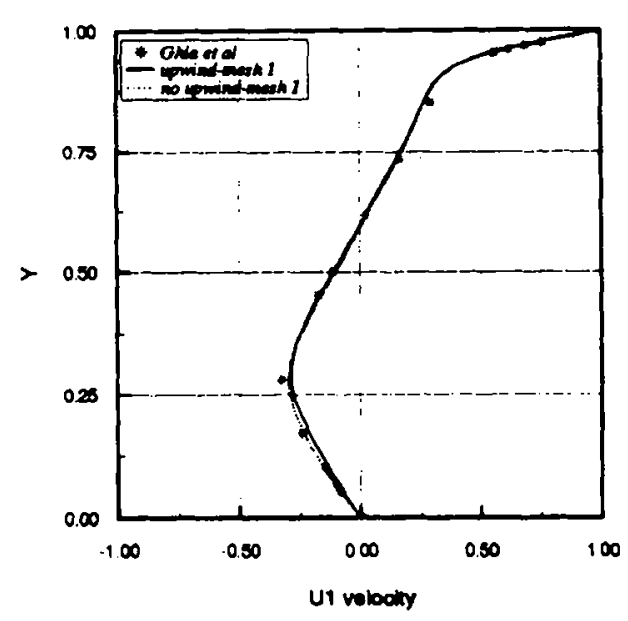

(a) $\operatorname{Re}=400$

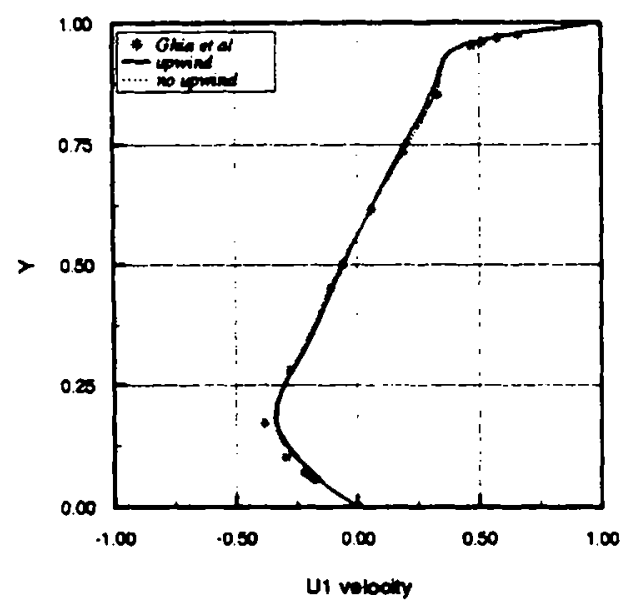

(b) $\operatorname{Re}=1000$

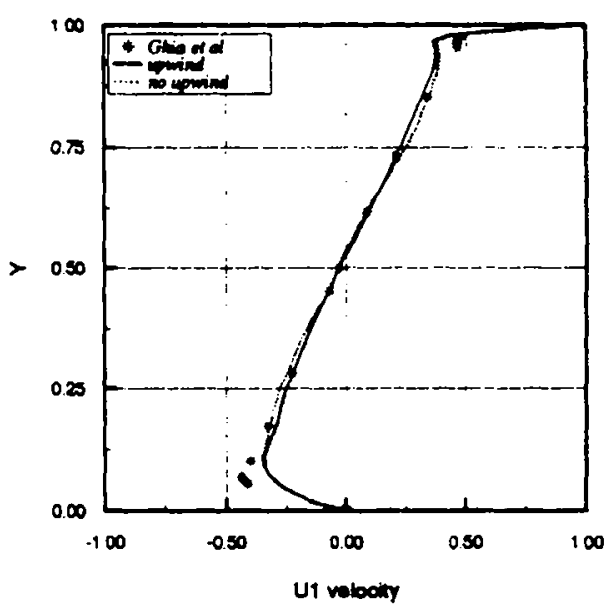

(c) $\operatorname{Re}=5000$

Figure 8. Comparison of $U_{1}$-velocity along mid-vertical plane with results of Ghia et al ${ }^{27}$ for various Reynolds numbers

number is taken as 3025 . A very fine mesh is required near the wall boundaries owing to the large velocity gradients present there. Figure 11 shows the linear triangular element mesh used for this problem. It consists of 2714 nodes and 5212 elements. The nearest node from the wall is taken at a distance of $y^{+}=0.7$, where

$$
y^{+}=\frac{y}{v} \sqrt{\left(\frac{\tau_{0}}{\rho}\right)} .
$$

Here $y$ is the normal distance from the nearest wall and $\tau_{\mathrm{o}}$ is the wall shear stress. The same boundary conditions as in Figure 1(a) are applied for velocities and pressure, except that a turbulent velocity profile is prescribed at the inlet. $\kappa=0$ is prescribed on all the walls, while at the inlet a turbulent 


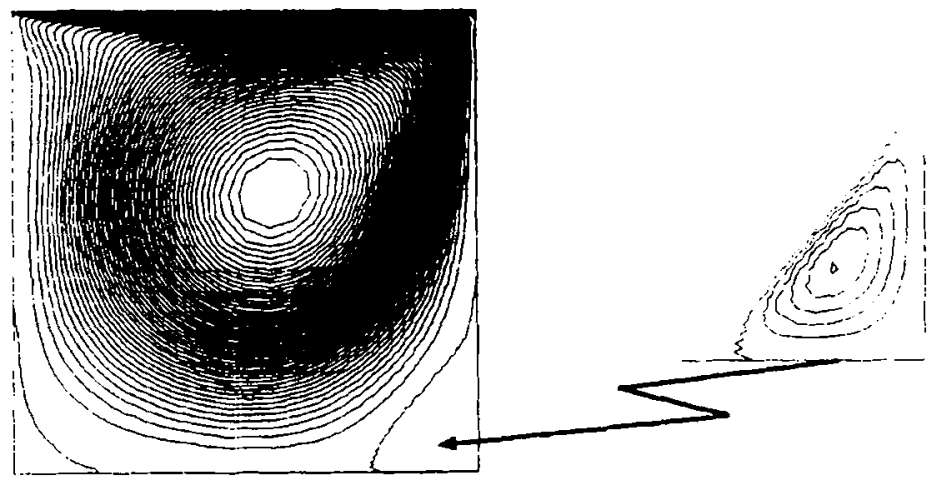

(a)

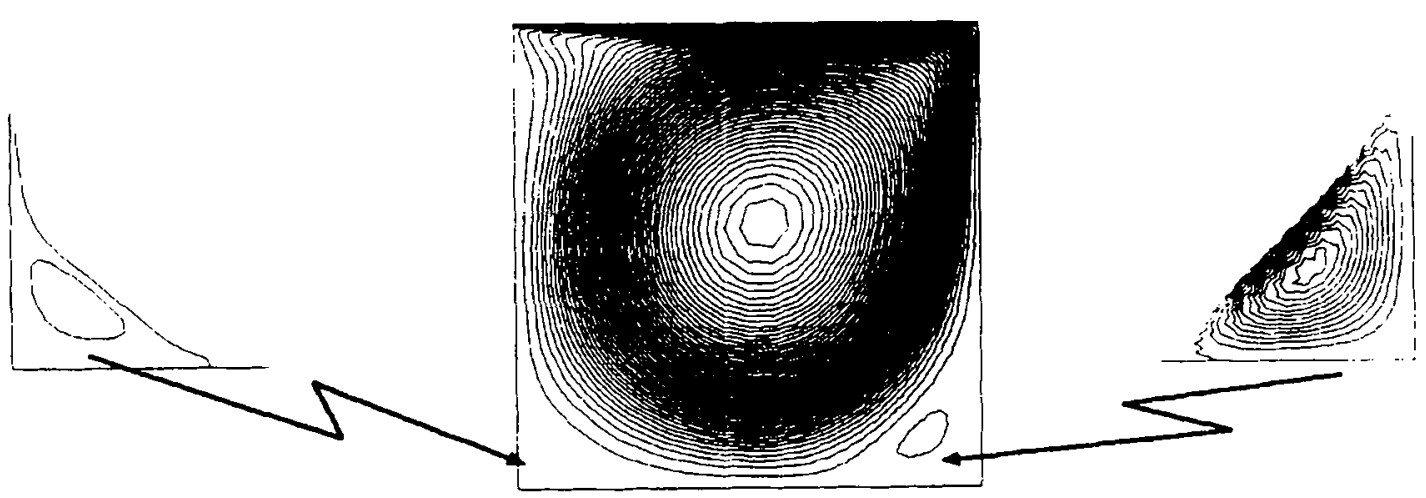

(b)
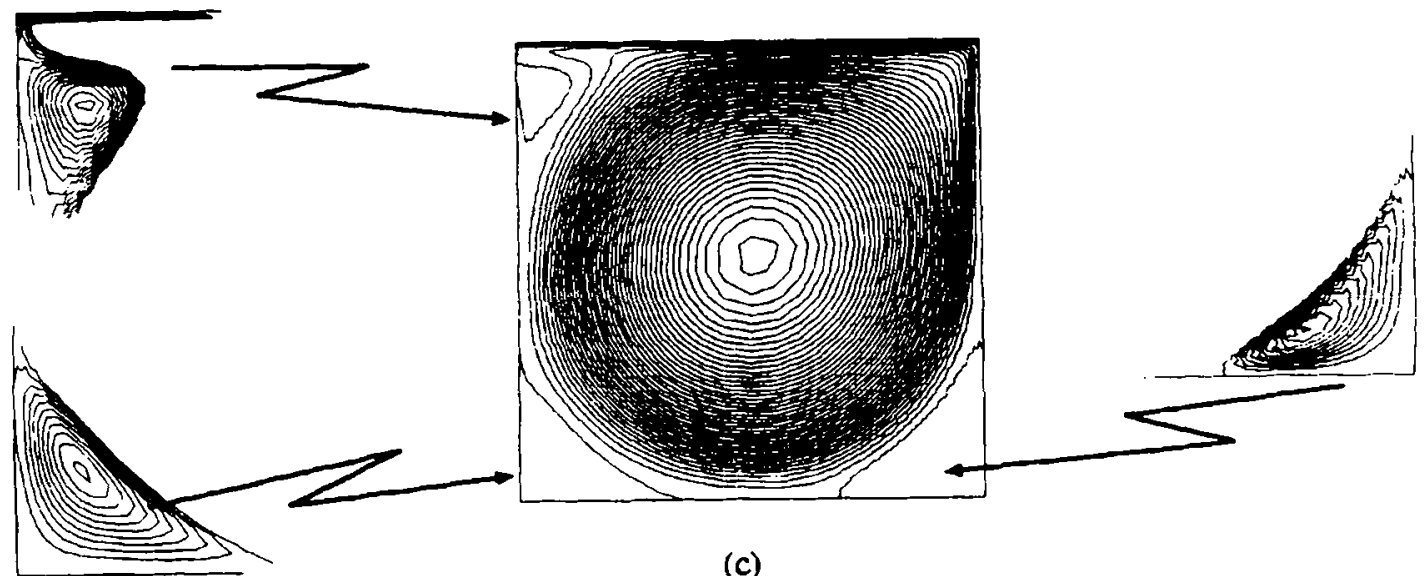

(c)

Figure 9. Streamlines for lid-driven cavity problem using present scheme: (a) $R e=400$; (b) $\operatorname{Re}=1000$; (c) $R e=5000$ (insets show streamlines in secondary circulation zones with smaller contour intervals) 


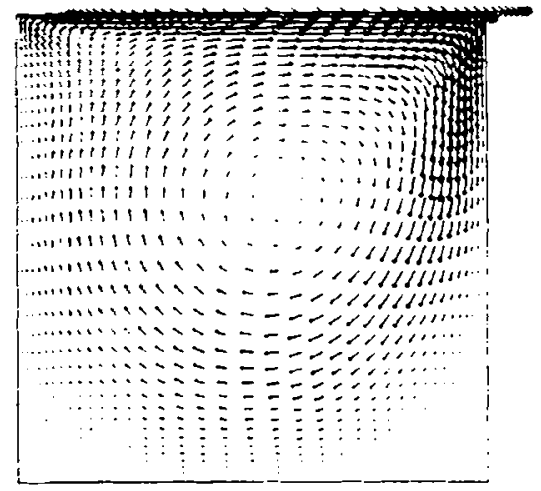

(a) $\operatorname{Re}=400$

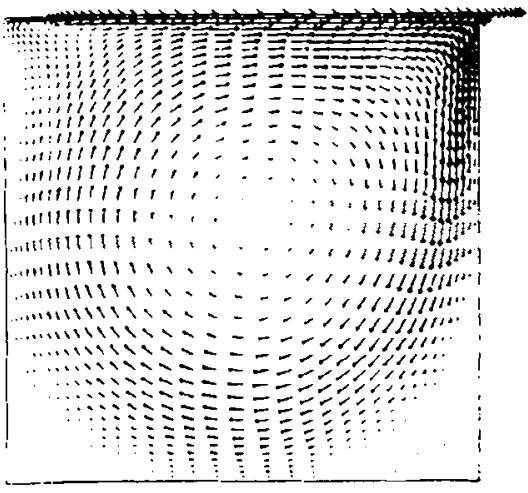

(b) $\operatorname{Re}=1000$

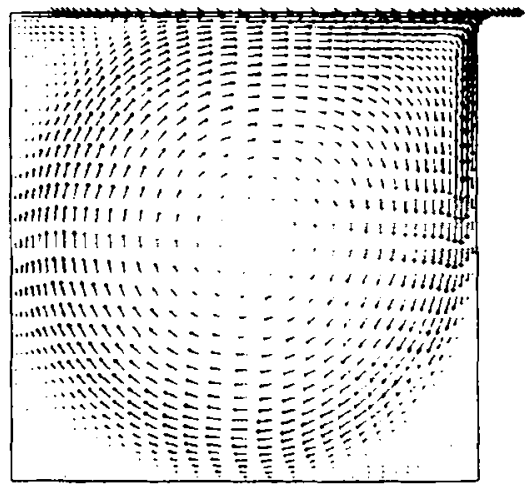

(c) $\operatorname{Re}=5000$

Figure 10. Velocity vectors for lid-driven cavity problem using present scheme

kinetic energy profile is prescribed. The velocity and turbulent kinetic energy profiles for the inlet are obtained from a one equation model analysis of turbulent flow through a channel of width same as at upstream of the step. Figure 12(a) shows the mesh for channel flow with $R e=3025$. Freestream conditions are prescribed at the inlet for velocities and turbulent kinetic energy. The fully developed profiles for velocity and turbulent kinetic energy obtained at the outlet are shown in Figures 12(b) and 12(c) respectively. These profiles are used at the inlet for both one and two equation model analyses for the step. In addition, for the two equation model analysis an inlet $\varepsilon$-profile as obtained from the channel analysis is applied. On all the walls the condition $\partial \epsilon / \partial n=0$ is applied. This is compatible with the damping functions used in the present analysis. It has been observed that for turbulent flows the present scheme does not result in an overdamping of flow quantities such as velocities and turbulent kinetic energy as was noted by some earlier investigators. ${ }^{20.26}$ This is due to the formula used for evaluating $P e$ (see equation (44)). In the mean turbulent flow where there are higher velocities, the turbulent viscosity is also relatively high. This gives a smaller value of $P e$, which in turn gives a smaller interior $\Delta t$. Thus very little damping is added in turbulent flow 


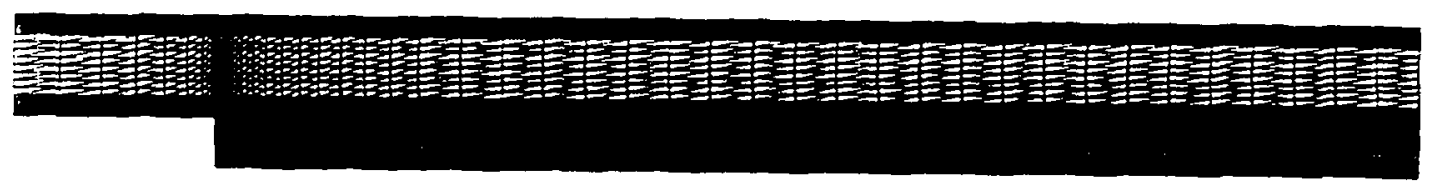

Figure 11. Mesh (2714 nodes, 5212 elements) for turbulent flow past a backward facing step

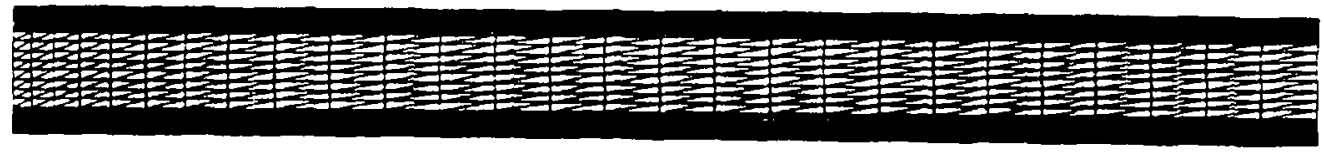

(a) Mesh, 1036 nodes, 1944 elements

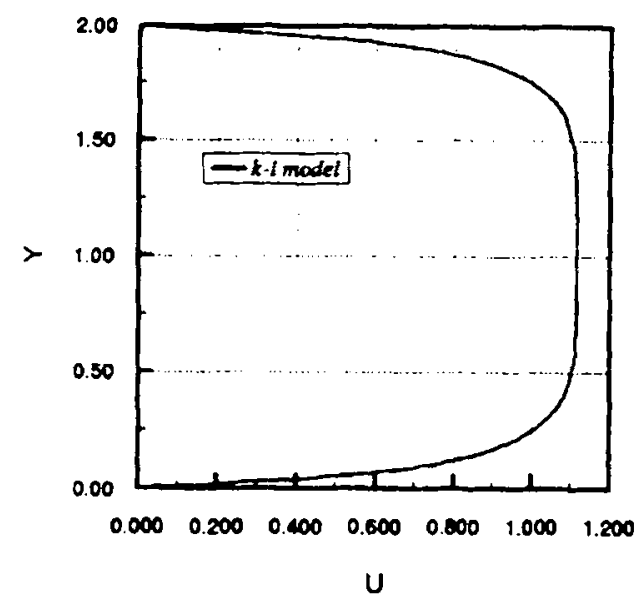

(b) Velocity profile at the exit

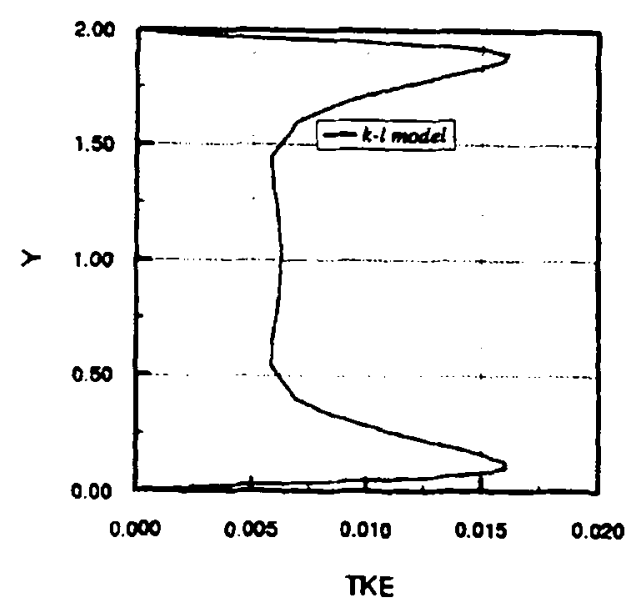

(c) Turbulent kinetic energy at the exit

Figure 12. Turbulent flow in a constant section channel, $k-t$ model

situations. For the one equation model, Figures 13(a) and 13(b) show the velocity and turbulence intensity profiles respectively at various sections downstream of the step compared with the experimental profiles obtained by Denham et al. ${ }^{31}$ Except for a small region in the circulation zone, in general there seems to be good agreement between the two. Figure 13(c) shows the streamline pattern for this case. Also shown is an enlarged view of the streamline pattern near the step. The reattachment length predicted by this model is $5.6 h-5.7 h$ as compared with $0.6 h$ by experiments. Here $h$ is the step height. The corresponding predictions by Taylor et al. ${ }^{26}$ and Atkins et al. ${ }^{20}$ are $5 \cdot 6 h$ and $5 \cdot 2 h$ respectively. Figure 14 shows similar plots obtained from the present scheme by applying the two equation model of turbulence. The significant differences observed are in the recirculation zone, where the turbulent kinetic energy prediction of the one equation model is slightly better than 


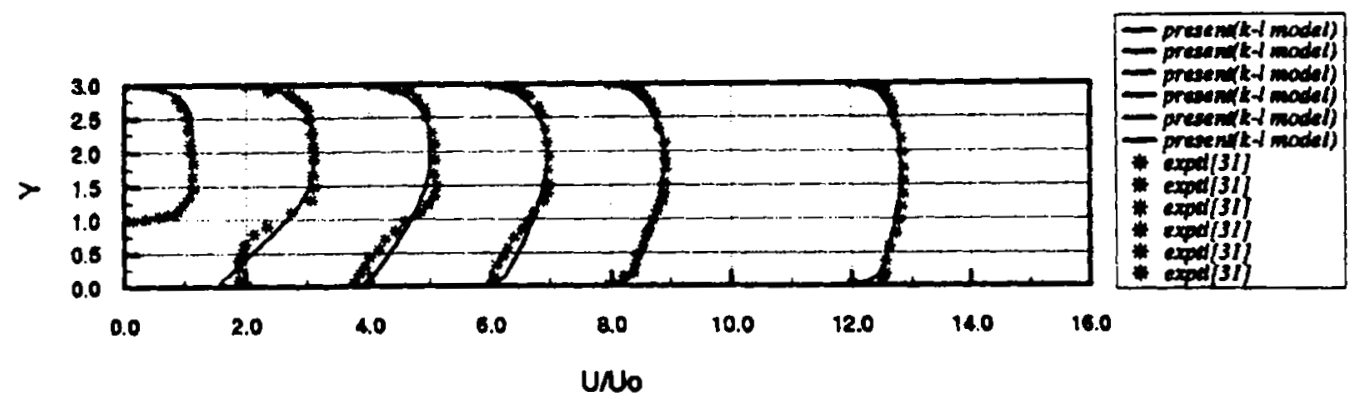

1 unit of $U / U_{0}=1$ unit of $X / h$

(a) Velocity profiles downward of the step

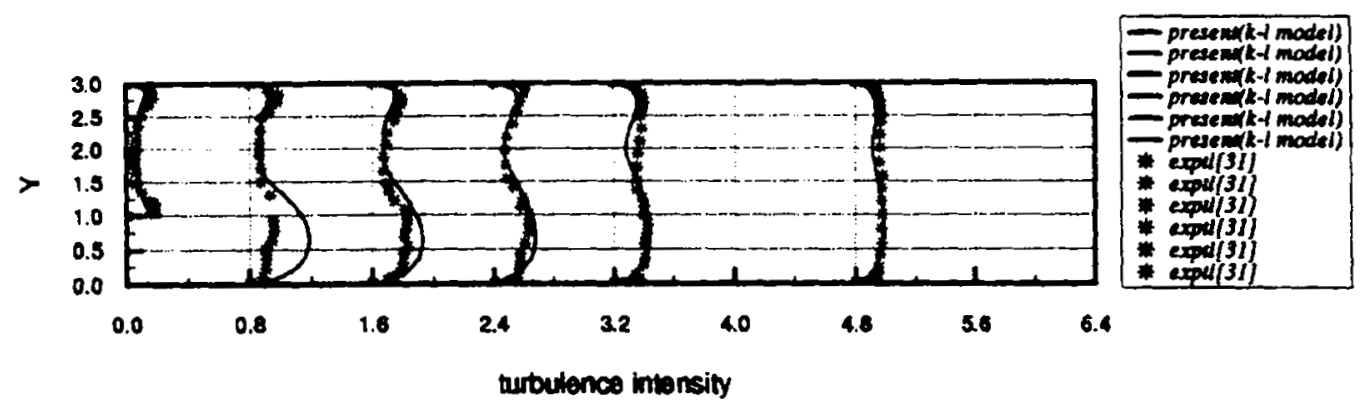

1 unit of T.I. $=2.5$ units of $X / h$

(b) Turbulence intensity profiles downward of the step

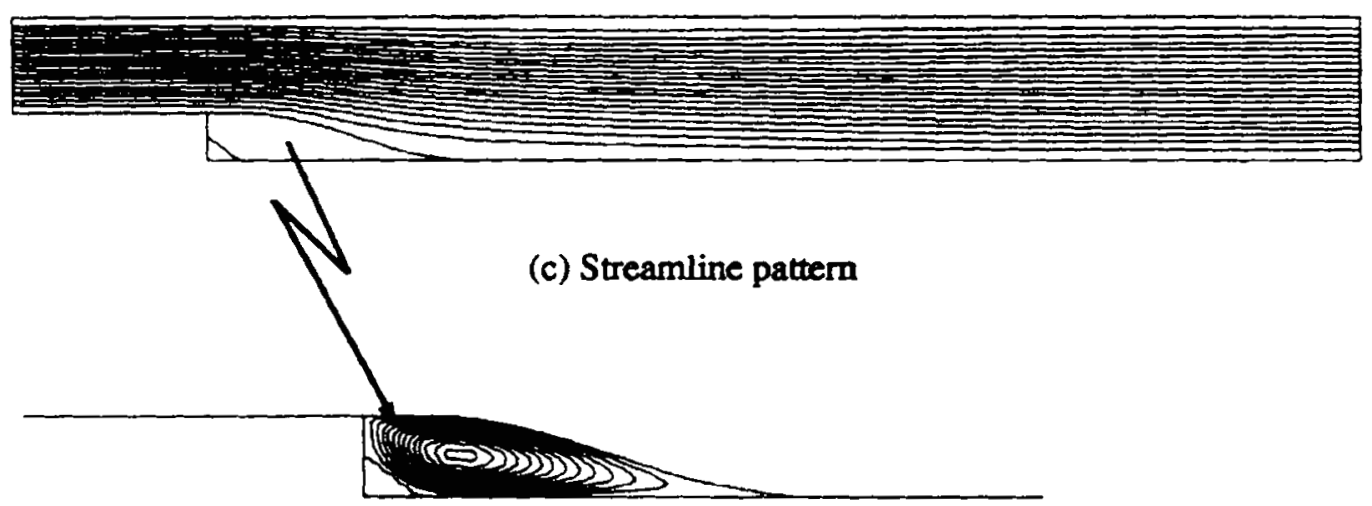

Figure 13. Results for one equation model of turbulence for $R e=3025$ using present scheme 


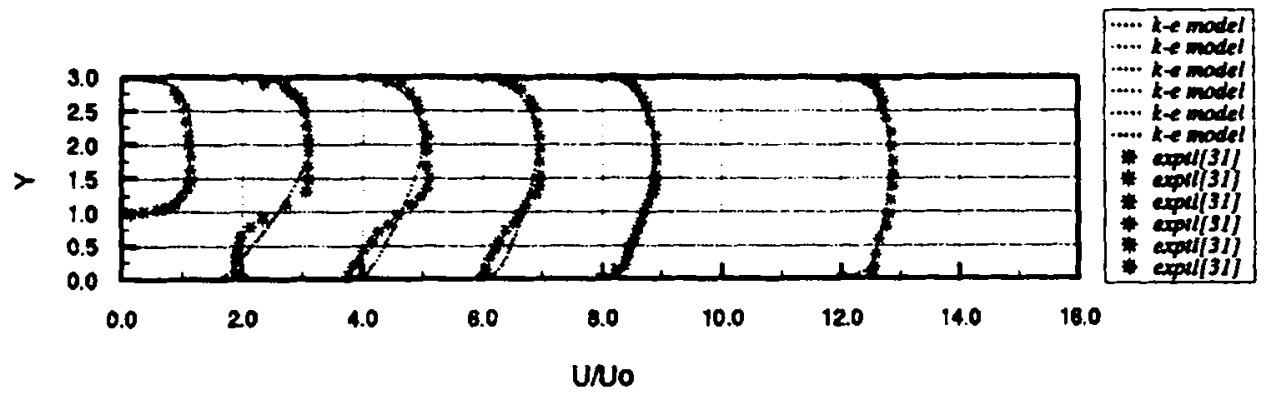

1 unit of $U / U_{0}=1$ unit of $X / h$

(a) Velocity profiles downward of the step

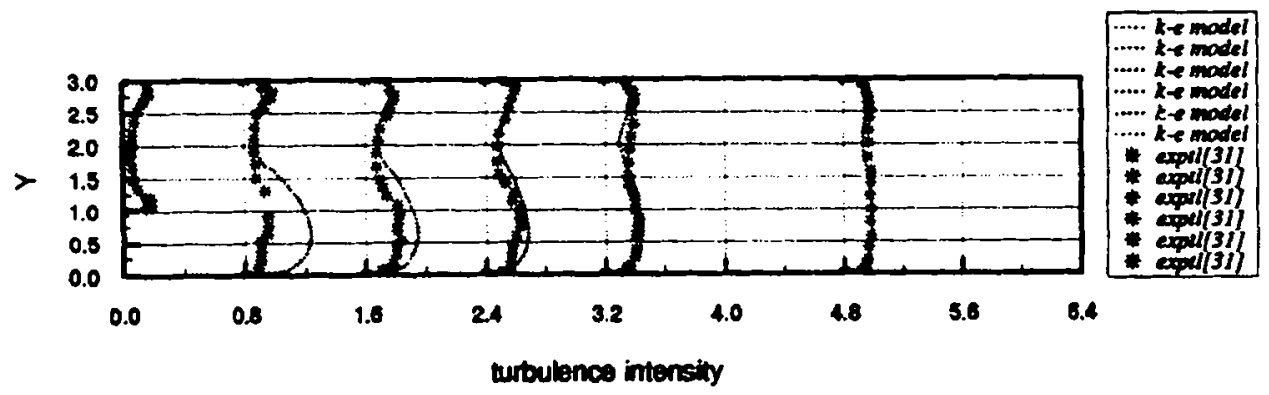

1 unit of T.I. $=2.5$ units of $X / \mathrm{h}$

(b) Turbulence intensity profiles downward of the step

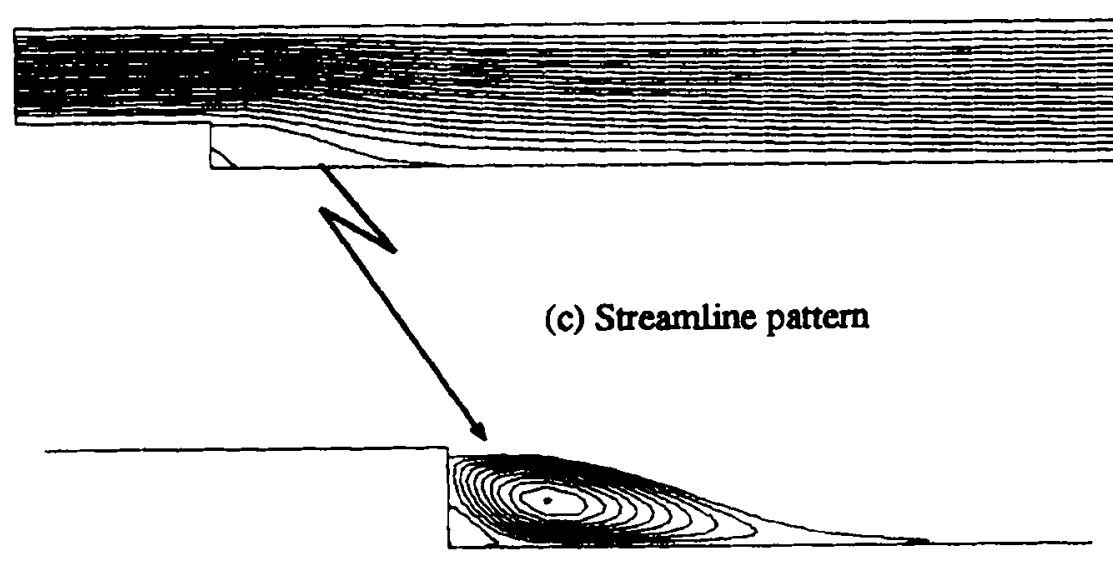

Figure 14. Results for two equation model of turbulence for $R e=3025$ using present scheme 
that of the two-equation model. Also, the reattachment length for the two-equation model is $4.7 \mathrm{~h}-$ $4.8 h$ as compared with $5 \cdot 6 h-5.7 h$ for the one-equation model. The corresponding predictions by Taylor et $a l^{26}$ and Atkins et $a .^{20}$ are $4.5 h$ and $4.2 h$ respectively. Similar observations about the poorer performance of the two- equation model compared with the one-equation model for the backward-facing step problem were made by several investigators. Thus this section shows the applicability of the present scheme in making turbulent flow predictions.

\section{CONCLUSIONS}

The general fluid mechanics algorithm originally presented in Reference 19 has been applied in its semi-implicit form to laminar and turbulent flow situations. Satisfactory predictions are obtained both for laminar flow over a backward-facing step and for the lid-driven cavity problem. The scheme has also been extended for turbulent flow situations incorporating both one- and two-equation models. Satisfactory predictions of reattachment lengths were made with the present scheme. By using the modification for the Peclet number calculation, it is possible to avoid the overdamping effect of upwinding for turbulent flow problems.

\section{REFERENCES}

1. P. Hood and C. Taylor, 'Navier-Stokes equations using mixed interpolation', in J. T. Oden et al. (eds), Finite Element Methods in Flow Problems, UAH Press, Huntsville, AL, 1974, pp. 121-132.

2. O. C. Zienkiewicz and R. L. Taylor, The Finite Element Method, 4th edn, McGraw-Hill, New York, 1989.

3. T. J. R. Hughes, L. P. Franca and M. Balastra, 'A new finite element formulation for computational fluid dynamics: V. Circumventing the Babüska-Brezzi condition: a stable Petrov-Galerkin formulation of the Stokes problems accommodating equal-order interpolations', Comput. Methods Appl. Mech. Eng., 59, 85-99 (1986).

4. R. L. Sani, P. M. Gresho, R. L. Lee and D. F. Griffiths, 'The cause and cure(?) of the spurious pressure generated by certain FEM solutions of the incompressible Navier-Stokes equations: Part I', int. j. numer. methods fuids, 1, 17-43 (1981).

5. R. L. Sani, P. M. Gresho, R. L. Lee, D. F. Griffiths and M. Engleman, 'The cause and cure(!) of the spurious pressure generated by certain FEM solutions of the incompressible Navier-Stokes equations: Part II', Int. $j$. numer. methods fiuids, 1, 171-204 (I981).

6. A. J. Chorin, 'Numerical solution of the Navier-Stokes equations', Math. Comput., 23, 341-354 (1968).

7. G. E. Schneider, G. D. Raithby and M. M. Yovanovich, 'Finite element analysis of incompressible fluid flow incorporating equal order pressure and velocity interpolation', in C. Taylor, K. Morgan and C. A. Brebbia (eds), Numerical Methods in Laminar and Turbulent Flow, Pentech, 1978.

8. M. Kawahara and K. Ohmiya, 'Finite element analysis of density flow using the velocity correction method', Int. $j$. numer. methods fluids, 5, 308-323 (1985).

9. B. Ramaswamy, 'Finite element solution for advection and natural convection flows', Comput. Fluids, 16, 349-388 (1988).

10. O. C. Zienkiewicz and J. Wu, 'A general explicit or semi-explicit algorithm for compressible or incompressible flows', Int. j. numer. methods eng., 35, 457-459 (1992).

11. O. C. Zienkiewicz and J. Wu, 'Incompressibility without tears-how to avoid the restrictions on mixed formulation', Int. $j$. numer. methods eng., 32, 1189-1203 (1991).

12. P. Gresho and R. L. Lee, 'Don't suppress wiggles they are telling you something', Comput. Fluids, 9, $223-253$ (1981).

13. I. Christie, D. F. Griffiths, A. R. Mitchell and O. C. Zienkiewicz, 'Finite element methods for second-order differential equations with significant first-order derivatives', Int. j. numer. methods eng., 10, 1389-1396 (1976).

14. J. C. Heinrich, P. S. Huyakom, A. R. Mitchell and O. C. Zienkiewicz, 'An upwind finite element scheme for two dimensional convective transport equations', Int. $j$. numer. methods eng., 11, $131-144$ (1977).

15. T. J. R. Hughes and A. N. Brooks, 'A multi-dimensional upwind scheme with no cross wind diffusion', in T. J. R. Hughes (ed.), Finite Elements for Convection Dominated Flows, AMD Vol. 34, ASME, New York, 1979.

16. D. N. Kelly, S. Nakazawa, O. C. Zienkiewicz and J. C. Heinrich, 'A note on anisotropic balancing dissipation in finite element method approximation to convection diffusion problems', Int. $j$. numer. methods eng., 15, 1705-1711 (1980).

17. C. Johnson, U. Navert and J. Pitkaranta, 'Finite element method for linear hyperbolic problems', Comput. Methods Appl. Mech. Eng., 45, 288-312 (1984).

18. R. Lohner, K. Morgan and $O$. C. Zienkiewicz, 'The solution of non- linear hyperbolic equation systems by the finite element method', Int. j. numer. methods fluids, 4, 1043-1063 (1984).

19. O. C. Zienkiewicz and R. Codina, 'A general algorithm for compressible and incompressible flow, Part 1: The split, characteristic-based scheme', Int. $j$. numer. methods fuids, 20, 869-885 (1995).

20. D. J. Atkins, S. J. Maskell and M. A. Patrick, 'Numerical prediction on separated flows', Int. j. numer. methods eng., 15, $129-144(1980)$. 
21. W. Rodi, Turbulence Models and Their Applications in Hydraulics- A State of the Art Review, IAHR.

22. M. Wolfstein, 'Some solutions of plane nurbulent impinging jets', ASME J. Basic Eng., 92, 915-922 (1970).

23. C. K. G. Lam and K. Bermhorst, 'A modified form of $k \in$ model for predicting wall turbulence', $J$. Fluids Eng., 103, 456$460(1981)$

24. R. Codina, M. Vázquez and O. C. Zienkiewicz, 'A fractional step method for compressible flows: boundary conditions and incompressible limit', Proc. Int. Conf. on Finite Elements in Fluids-New Trends and Applications, Venice, October 1995, pp. $409-418$.

25. M. K. Denham and M. A. Patrick, 'Laminar flow over a downstream- facing step in a two-dimensional flow channel', Trans. Inst. Chem. Eng., 52(4), 361-367 (1974).

26. C. Taylor, C. E. Thomas and K. Morgan, 'Analysis of turbulent flow with separation using the finite element method', in C. Taylor and K. Morgan (eds), Recent Advances in Numerical Methods in Fluids, Vol. 2, Computational Techniques in Transient and Turbulent Flow, Pineridge, Swansea, 1981, pp. 283-325

27. U. Ghia, K. N. Ghia and C. T. Shin, 'High-Re solutions for incompressible flow using the Navier-Stokes equations and a multi-grid', J. Comput. Phys., 48, 387-411 (1982).

28. J. L. Sohn, 'Evaluation of FIDAP on some classical laminar and nurbulent benchmarks', Int. j. numer. methods fluids, 8 , 1469-1490 (1988)

29. L. P. Hackman, G. D. Raithby and A. B. Strong, 'Numerical predictions of flows over backward facing steps', Int. j. numer. methods fluids, 4, 711-724 (1984).

30. A. Autret, M. Grandotto and 1. Dekeyser, 'Finite element computation of a turbulent flow over a two-dimensional backward-facing step', Int. j. numer. methods fuids, 7, 89-102 (1987).

31. M. K. Denham, P. Briard and M. A. Patrick, 'A directionally sensitive laser anemometer for velocity measurements in highly turbulent flow', J. Phys. E: Sci. Instrum., 8, 681-683 (1975).

32. O. C. Zienkiewicz, K. Morgan, B. V. K. Satya Sai, R. Codina and M. Vázquez, 'A general algorithm for compressible and incompressible flow, Part II: Tests on the explicit form', Int. $j$. numer. methods fuids, 20, 887-913 (1995). 